\title{
Predictors of COVID-19 severity: a systematic review and
}

\section{meta-analysis [version 1; peer review: 2 approved]}

\author{
Mudatsir Mudatsir (D1, Jonny Karunia Fajar (D1,2, Laksmi Wulandari3, \\ Gatot Soegiarto (D)4, Muhammad Ilmawan (D5), Yeni Purnamasari (D) \\ Bagus Aulia Mahdi (D) 4, Galih Dwi Jayanto², Suhendra Suhendra5, \\ Yennie Ayu Setianingsih (D)6, Romi Hamdani7, Daniel Alexander Suseno (iD), \\ Kartika Agustina9, Hamdan Yuwafi Naim (D10, Muchamad Muchlas (D11, \\ Hamid Hunaif Dhofi Alluza5, Nikma Alfi Rosida5, Mayasari Mayasari5, \\ Mustofa Mustofa5, Adam Hartono12, Richi Aditya(iD) 5irman Prastiwi(i)5, \\ Fransiskus Xaverius Meku5, Monika Sitio5, Abdullah Azmy7 , Anita Surya Santoso ${ }^{13}$, \\ Radhitio Adi Nugroho5, Camoya Gersom², Ali A. Rabaan (D14, Sri Masyeni (i)15, \\ Firzan Nainu(iD16, Abram L. Wagner (Di)17, Kuldeep Dhama (Di)18, \\ Harapan Harapan (D)1,19
}

\footnotetext{
${ }^{1}$ Faculty of Medicine, Universitas Negeri Sebelas Maret, Surakarta, Surakarta, 57126, Indonesia

${ }^{2}$ Department of Cardiology and Vascular Medicine, Faculty of Medicine, Universitas Brawijaya, Malang, East Java, 65145, Indonesia

${ }^{3}$ Molecular Diagnostic Laboratory, Johns Hopkins Aramco Healthcare, Dhahran, Dhahran, 31311, Saudi Arabia

${ }^{4}$ Department of Internal Medicine, Faculty of Medicine and Health Science, Universitas Warmadewa, Denpasar, Bali, 80235 , Indonesia

5 Faculty of Pharmacy, Hasanuddin University, Makassar, Makassar, 90245, Indonesia

${ }^{6}$ Department of Epidemiology, University of Michigan, Ann Arbor, MI, 48109, USA

${ }^{7}$ Division of Pathology, Indian Veterinary Research Institute, Izatnagar, Uttar Pradesh, 243 122, India

${ }^{8}$ Medical Research Unit, School of Medicine, Universitas Syiah Kuala, Banda Aceh, Aceh, 23111, Indonesia

${ }^{9}$ Department of Microbiology, School of Medicine, Syiah Kuala University, Banda Aceh, Aceh, 23111, Indonesia

${ }^{10}$ Brawijaya Internal Medicine Research Center, Department of Internal Medicine, Faculty of Medicine, Universitas Brawijaya,

Malang, East Java, 65145, Indonesia

${ }^{11}$ Department of Pulmonology and Respiratory Medicine, Faculty of Medicine, Universitas Airlangga, Surabaya, East Java, 60286, Indonesia

${ }^{12}$ Department of Internal Medicine, Faculty of Medicine, Universitas Airlangga, Surabaya, Easy Java, 60286, Indonesia

${ }^{13}$ Faculty of Medicine, Universitas Brawijaya, Malang, East Java, 65145, Indonesia

${ }^{14}$ Department of Urology, Faculty of Medicine, Universitas Airlangga, Surabaya, East Java, 60285, Indonesia

${ }^{15}$ Department of Orthopedic Surgery, Faculty of Medicine, Universitas Brawijaya, Malang, East Java, 65145, Indonesia

${ }^{16}$ Department of Obstetry and Gynecology, Faculty of Medicine, Universitas Brawijaya, Malang, East Java, 65145, Indonesia

${ }^{17}$ Department of Neurology, Faculty of Medicine, Universitas Brawijaya, Malang, East Java, 65145, Indonesia

${ }^{18}$ Department of Urology, Faculty of Medicine, Universitas Brawijaya, Malang, East Java, 65145, Indonesia

${ }^{19}$ Faculty of Animal Science, Universitas Brawijaya, Malang, East Java, 65145, Indonesia
}

V1 First published: 09 Sep 2020, 9:1107

https://doi.org/10.12688/f1000research.26186.1

Latest published: 06 Jan 2021, 9:1107

https://doi.org/10.12688/f1000research.26186.2
Open Peer Review

Approval Status 


\section{Abstract}

Background: The unpredictability of the progression of coronavirus disease 2019 (COVID-19) may be attributed to the low precision of the tools used to predict the prognosis of this disease.

Objective: To identify the predictors associated with poor clinical outcomes in patients with COVID-19.

Methods: Relevant articles from PubMed, Embase, Cochrane, and Web of Science were searched and extracted as of April 5, 2020. Data of interest were collected and evaluated for their compatibility for the meta-analysis. Cumulative calculations to determine the correlation and effect estimates were performed using the $Z$ test.

Results: In total, 19 papers recording 1,934 mild and 1,644 severe cases of COVID-19 were included. Based on the initial evaluation, 62 potential risk factors were identified for the meta-analysis. Several comorbidities, including chronic respiratory disease, cardiovascular disease, diabetes mellitus, and hypertension were observed more frequent among patients with severe COVID-19 than with the mild ones. Compared to the mild form, severe COVID-19 was associated with symptoms such as dyspnea, anorexia, fatigue, increased respiratory rate, and high systolic blood pressure. Lower levels of lymphocytes and hemoglobin; elevated levels of leukocytes, aspartate aminotransferase, alanine aminotransferase, blood creatinine, blood urea nitrogen, high-sensitivity troponin, creatine kinase, highsensitivity C-reactive protein, interleukin 6, D-dimer, ferritin, lactate dehydrogenase, and procalcitonin; and a high erythrocyte sedimentation rate were also associated with severe COVID-19.

Conclusion: More than 30 risk factors are associated with a higher risk of severe COVID-19. These may serve as useful baseline parameters in the development of prediction tools for COVID-19 prognosis.

\section{Keywords}

SARS-CoV-2, COVID-19, prognosis, severity, clinical outcome

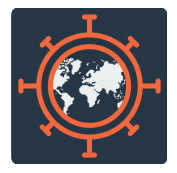

This article is included in the Emerging Diseases and Outbreaks gateway.

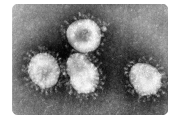

This article is included in the Coronavirus collection.

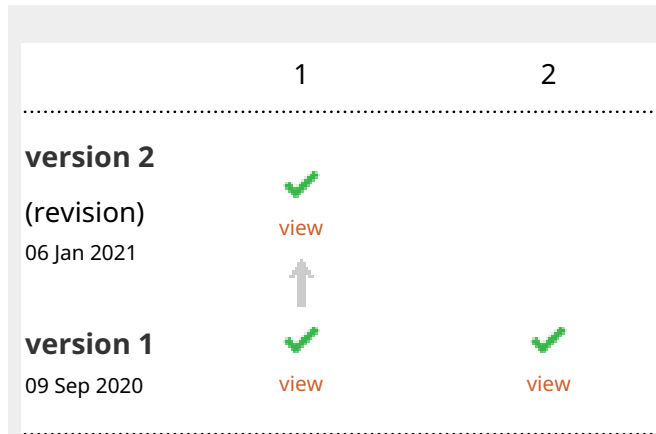

1. Morteza Arab-Zozani (D), Birjand University of Medical Sciences, Birjand, Iran

2. Annelies Wilder-Smith, University of Heidelberg, Heidelberg, Germany Any reports and responses or comments on the article can be found at the end of the article. 
Corresponding authors: Mudatsir Mudatsir (mudatsir@unsyiah.ac.id), Laksmi Wulandari (laksmi.wulandari@fk.unair.ac.id)

Author roles: Mudatsir M: Conceptualization, Formal Analysis, Investigation, Methodology, Project Administration, Supervision, Validation, Writing - Original Draft Preparation, Writing - Review \& Editing; Fajar JK: Conceptualization, Formal Analysis, Investigation, Methodology, Software, Supervision, Validation, Writing - Original Draft Preparation, Writing - Review \& Editing; Wulandari L: Formal Analysis, Investigation, Methodology, Project Administration, Supervision, Validation, Writing - Original Draft Preparation; Soegiarto G: Conceptualization, Investigation, Methodology, Validation, Writing - Original Draft Preparation; Ilmawan M: Conceptualization, Formal Analysis, Investigation, Methodology, Project Administration, Software, Validation, Writing - Original Draft Preparation; Purnamasari Y: Formal Analysis, Investigation, Methodology, Project Administration, Resources, Software, Supervision, Validation, Visualization, Writing - Original Draft Preparation; Mahdi BA: Conceptualization, Formal Analysis, Investigation, Methodology, Project Administration, Resources, Software, Validation, Writing - Original Draft Preparation; Jayanto GD: Conceptualization, Formal Analysis, Investigation, Methodology, Resources, Software, Supervision, Writing - Original Draft Preparation; Suhendra S: Data Curation, Investigation, Methodology, Resources, Software, Visualization; Setianingsih YA: Conceptualization, Formal Analysis, Investigation, Methodology, Resources, Software, Validation; Hamdani R: Funding Acquisition, Investigation, Methodology, Resources, Software; Suseno DA: Data Curation, Investigation, Methodology, Resources, Software; Agustina K: Data Curation, Investigation, Methodology, Resources, Software; Naim HY: Formal Analysis, Investigation, Methodology, Project Administration, Resources, Software; Muchlas M: Data Curation, Investigation, Methodology, Project Administration, Resources, Software; Alluza HHD: Data Curation, Formal Analysis, Investigation, Methodology, Project Administration, Resources, Software; Rosida NA: Data Curation, Formal Analysis, Investigation, Methodology, Resources, Software; Mayasari M: Data Curation, Formal Analysis, Investigation, Methodology, Resources, Software; Mustofa M: Data Curation, Formal Analysis, Investigation, Methodology, Resources; Hartono A: Data Curation, Formal Analysis, Investigation, Methodology, Software; Aditya R: Data Curation, Formal Analysis, Investigation, Methodology, Project Administration, Resources, Software; Prastiwi F: Data Curation, Investigation, Methodology, Resources, Software; Meku FX: Data Curation, Investigation, Methodology, Project Administration, Resources, Software; Sitio M: Data Curation, Investigation, Methodology, Project Administration, Resources, Software; Azmy A: Data Curation, Investigation, Methodology, Resources, Software; Santoso AS: Data Curation, Funding Acquisition, Investigation, Methodology, Project Administration, Resources, Software; Nugroho RA: Data Curation, Funding Acquisition, Investigation, Methodology, Resources, Software; Gersom C: Data Curation, Formal Analysis, Investigation, Methodology, Resources, Software; Rabaan AA: Data Curation, Investigation, Methodology, Resources, Software; Masyeni S: Data Curation, Investigation, Methodology, Resources, Software; Nainu F: Data Curation, Investigation, Methodology, Resources, Software; Wagner AL: Data Curation, Formal Analysis, Investigation, Methodology, Resources, Software, Supervision, Validation, Writing - Review \& Editing; Dhama K: Supervision, Validation, Visualization, Writing - Review \& Editing; Harapan H: Data Curation, Formal Analysis, Investigation, Methodology, Project Administration, Supervision, Validation, Visualization, Writing - Original Draft Preparation, Writing Review \& Editing

Competing interests: No competing interests were disclosed.

Grant information: We thank to Lembaga Pengelola Dana Pendidikan (LPDP) Republik Indonesia for supporting this project. The funders had no role in study design, data collection and analysis, decision to publish, or preparation of the manuscript.

Copyright: @ 2020 Mudatsir $\mathrm{M}$ et al. This is an open access article distributed under the terms of the Creative Commons Attribution License, which permits unrestricted use, distribution, and reproduction in any medium, provided the original work is properly cited.

How to cite this article: Mudatsir M, Fajar JK, Wulandari L et al. Predictors of COVID-19 severity: a systematic review and metaanalysis [version 1; peer review: 2 approved] F1000Research 2020, 9:1107 https://doi.org/10.12688/f1000research.26186.1

First published: 09 Sep 2020, 9:1107 https://doi.org/10.12688/f1000research.26186.1 


\section{Introduction}

The coronavirus disease 2019 (COVID-19) pandemic, caused by severe acute respiratory syndrome coronavirus 2 (SARS-CoV-2), is a global crisis across health, economic, and educational dimensions ${ }^{1,2}$. The disease has spread rapidly, can cause severe illness, and is characterized by a high mortality rate in certain groups. Mortality is particularly high in the absence of proven effective standard management measures ${ }^{3}$. One of the problems with the management of this disease is the absence of standardized methods for diagnosis and the inability to estimate prognosis based on clinical features. Certain reports have shown that poor prognostic prediction has correlated with high mortality among patients with COVID-19 $9^{4,5}$. Among patients with similar clinical characteristics and with similar treatment regiments, there may be a diversity in clinical outcomes ${ }^{6}$. Therefore, the development and use of an accurate predictor for COVID-19 prognosis will be beneficial for the clinical management of patients with COVID-19, and will help reduce the mortality rate. Successful implementation of such a prediction mechanism could have a large public health impact. Better understanding of clinical progression could also improve public health messaging, particularly as many individuals may consider COVID-19 to not be severe.

Prognostic tools for the prediction of COVID-19 severity in patients have been in development since January 2020. At least nine studies proposed the use of prognostic tools for the prediction of COVID-19 severity ${ }^{7-15}$. However, a recent systematic review and critical appraisal study evaluated the accuracy of these tools using prediction model risk of bias assessment tool (PROBAST) and reported a high risk of bias ${ }^{16}$. The establishment of a prediction model for the estimation of disease prognosis may help health workers segregate patients according to prediction status. However, the high risk of bias in these prediction tools might lead to inaccurate prediction of COVID19 severity. A comprehensive study of the identification of risk factors that might play a significant role in determining the severity of patients with COVID-19 is necessary. We performed a systematic review and meta-analysis to assess the risk factors associated with poor clinical outcomes among patients with COVID-19. To the best of our knowledge, this is the first meta-analysis to assess the comprehensive risk factors that might affect the severity of COVID-19 in patients. The results of our study might serve as preliminary data for the compilation or improvement of the scoring system in the prediction of COVID-19 severity.

\section{Methods}

Study design

We performed a systematic review and meta-analysis to evaluate potential risk factors that might influence the severity of COVID-19. These risk factors include comorbidities, clinical manifestations, and laboratory findings. Accordingly, we searched the relevant studies from major scientific websites and databases to collect the data of interest, and determined the association and effect estimates by calculating the combined odds ratio (OR) and $95 \%$ confidence intervals $(95 \% \mathrm{CI})$. The protocols for the systematic review and meta-analysis were similar to those used in previous studies ${ }^{17-23}$, as well as to those recommended by Preferred Reporting Items for Systematic Review and Meta-Analysis (PRISMA) ${ }^{24}$.

\section{Eligibility criteria}

Studies were included in this review if they met the following inclusion criteria: (1) assessed the clinical manifestations and laboratory findings of patients with mild to severe COVID-19; (2) provided adequate data for the calculation of OR and $95 \%$ CI. Review articles, articles with non-standard data presentation, and duplicate publications were excluded.

\section{Search strategy and data extraction}

Major scientific databases (PubMed, Embase, Cochrane, and Web of Science) were searched for articles as of April 5, 2020. A comprehensive initial search was performed to identify the potential predictors, and a final search was performed to identify the relevant papers that could be included in the meta-analysis. We used the keywords adapted from medical subject headings: ["COVID-19" or "Coronavirus disease-19" or "SARS-CoV-2"] and ["mild" or "severe" or "prognosis" or "clinical outcome"] and ["clinical manifestation" or "morbidity" or "laboratory findings"]. Only studies written in English were included. If a duplicate publication was found, the article with the larger sample size was included. We also searched for relevant studies from the reference lists in the articles. During data extraction, the following information of interest was extracted: (1) first author name; (2) publication year; (3) sample size of mild and severe cases, (4) clinical manifestations, (5) morbidities, and (6) laboratory findings. Data extraction was performed by two independent investigators (JKF and MI) using a pilot form.

\section{Assessment of the methodological quality}

Before inclusion in the meta-analysis, the methodological quality of the articles was assessed using the New Castle-Ottawa scale (NOS). NOS scores range from 0 to 9 and consider three items: selection of patients (4 points), comparability of the groups (2 points), and ascertainment of exposure (3 points). Each study was interpreted to be of low quality (for scores $\leq 4$ ), moderate quality (for scores between 5-6), or high quality (for scores $\geq 7)^{25}$. Articles with moderate to high quality were included in the analysis. The study assessment was conducted by two independent investigators (MI and YP) using a pilot form. The discrepancies between the findings of the two investigators were solved by consulting with another investigator (JKF).

\section{Study measures}

The outcome measure of the study was the severity of COVID-19 (mild vs. severe). The risk factors or predictors included three major groups: comorbidities, clinical manifestations, and laboratory parameters. Comorbid factors such as chronic kidney disease, chronic liver disease, chronic respiratory disease, cerebrovascular accident, cardiovascular disease, diabetes mellitus, hypertension, and malignancy were compatible with the analysis. For clinical manifestations, fever, cough, dry cough, expectoration, sore throat, dyspnea, diarrhea, myalgia, nasal 
congestion, anorexia, abdominal pain, fatigue, dizziness, headache, fever, heart rate, respiratory rate, systolic blood pressure, and diastolic blood pressure were included in this study. Among laboratory characteristics, the presence of leukocytosis, leukocytopenia, anemia, lymphocytopenia; the levels or the counts of white blood cell (WBC), hemoglobin, neutrophil, lymphocyte, monocyte, platelet, activated partial thromboplastin time (aPTT), partial thromboplastin time (PTT), aspartate aminotransferase (AST), alanine aminotransferase (ALT), total bilirubin, albumin, serum creatinine, blood urea nitrogen (BUN), high-sensitivity (Hs)-troponin I, creatine kinase, high-sensitivity C-reactive protein (Hs-CRP), C-reactive protein (CRP) >8 mg/L, interleukin 6 (IL-6), glucose, D-dimer, serum ferritin, sodium, potassium, lactate dehydrogenase, and procalcitonin, CD4 and CD8; erythrocyte sedimentation rate (ESR); elevated IL-16; and elevated ESR were all included.

\section{Statistical analysis}

The significant risk factors that might govern the severity of COVID-19 were determined by the calculation of a pooled OR and $95 \%$ CI. The significance of the pooled ORs was determined using the $\mathrm{Z}$ test $(\mathrm{p}<0.05$ was considered statistically significant). Prior to identification of the significant risk factors, data were evaluated for heterogeneity and potential publication bias. The heterogeneity among included studies was evaluated using the $\mathrm{Q}$ test. If heterogeneity existed $(\mathrm{p}<0.10)$, a random effect model was adopted; if not, a fixed effect model was adopted. Egger's test and a funnel plot were used to assess the reporting or publication bias $(\mathrm{p}<0.05$ was considered statistically significant). Furthermore, we performed a moderator analysis to identify the independent predictors of poor clinical outcomes among patients with COVID-19. The data were analyzed using Review Manager version 5.3 (Revman Cochrane, London, UK). To prevent analytical errors, statistical analysis was performed by two authors (JKF and MI). The cumulative calculation was presented in a forest plot.

\section{Results}

Eligible studies

Our searches yielded 6,209 potentially relevant studies, of which 6,170 studies were excluded after assessment of the titles and abstracts. Subsequently, further review of the complete texts was performed for 39 potential studies. In the full text review, we excluded 20 studies because they were reviews articles $(\mathrm{n}=9)$, inadequacy of data for the calculation of OR and $95 \%$ CI $(n=7)$, and poor quality $(n=4)$. Eventually, 19 papers were included in our meta-analysis ${ }^{26-42}$ The paper selection process adopted in our study is summarized in Figure 1, and the characteristics of studies included in our analysis are outlined in Table 1.

\section{Risk factors of severe COVID-19}

We found that eight comorbidities, 19 clinical manifestations, and 35 laboratory parameters were available for the metaanalysis (Table 2 and Table 3). Among the comorbid factors, chronic respiratory disease (OR: 2.48; 95\% CI: 1.44, 4.27), cardiovascular disease (OR: 1.70; 95\% CI: 1.05, 2.78), diabetes mellitus (OR: 2.10; 95\% CI: 1.33, 3.34), and hypertension (OR: 2.33 ; 95\% CI: $1.42,3.81)$ were associated with a greater risk of severe COVID-19 (Figure 2A-D).

Among the clinical manifestations, dyspnea (OR: 3.28; 95\% CI: $2.09,5.15)$, anorexia (OR: $1.83 ; 95 \% \mathrm{CI}: 1.00,3.34)$, fatigue (OR: 2.00; 95\% CI: 1.25, 3.20), and dizziness (OR: 2.67; 95\% CI: $1.18,6.01)$ were associated with severe COVID-19 (Figure 3A-D). In addition, increased respiratory rate (OR: 2.85; 95\% CI: 1.28, 6.33) and increased systolic blood pressure (OR: $1.84 ; 95 \%$ CI: $1.31,2.60$ ) were also associated with severe COVID-19 (Figure 4A and B). Compared to productive cough, dry cough was associated with a lower risk of severe COVID-19 (OR: 0.66; 95\% CI: 0.44, 0.97).

Among laboratory characteristics, severe COVID-19 was associated with elevated WBC count (OR: 4.92; 95\% CI: 2.12, 11.31), increased neutrophil count (OR: 5.45; 95\% CI: 2.04, 14.54), lymphocytopenia (OR: 3.19; 95\% CI: 1.14, 7.07), and decreased hemoglobin levels (OR: 0.76; 95\%CI: 0.58, 1.00) (Figure 5A-D). Elevated levels of AST, ALT, and serum creatinine increased the risk for severe manifestations of COVID-19 (ORs 4.91, 3.23, and 2.14, respectively; Figure 6A-C). Elevated levels of BUN (OR: 6.15; 95\% CI: 3.05, 12.37), Hs-troponin I (OR: 9.25; 95\% CI: 3.51, 24.37), creatine kinase (OR: 2.44; 95\% CI: 1.65, 3.62), Hs-CRP (OR: 14.27; 95\% CI: 5.13, 39.71), IL-6 (OR: 6.68; 95\% CI: 3.20, 13.94), D-dimer (OR: 6.19; 95\% CI: 4.22, 9.08), ferritin (OR: 1.96; 95\% CI: 1.06, 3.62), lactate dehydrogenase (OR: 8.28; 95\% CI: 4.75, 14.46), procalcitonin (OR: 6.62; 95\% CI: 3.32, 13.21), ESR (OR: 4.45; 95\% CI: 2.56, 7.76), and CRP >8 (OR: 8.34 ; 95\% CI: $1.85,37.62$ ) were also associated with severe COVID-19 (Figure 7-Figure 9). A low risk of severe COVID-19 was associated with low leukocyte levels (OR: 0.59 ; 95\% CI: $0.41,0.87$ ) and elevated lymphocyte levels (OR: 0.34; 95\% CI: 0.23, 0.50).

\section{Source of heterogeneity}

Heterogeneity was detected in the data of chronic kidney disease, cerebrovascular disease, cardiovascular disease, diabetes mellitus, hypertension, and malignancy among the comorbid factors analyzed. Therefore, we used the random effect model to analyze the data. The fixed effect model was used to analyze the data on chronic liver disease and chronic respiratory disease, as there was no evidence of heterogeneity. For clinical manifestations, the data on fever, cough, sore throat, dyspnea, diarrhea, anorexia, fatigue, temperature $>38^{\circ} \mathrm{C}$, respiratory rate, and diastolic blood pressure were analyzed using the random effect model while the rest of clinical manifestation data were analyzed using the fixed effect model.

Among laboratory parameters, evidence of heterogeneity was found in count of WBC, neutrophil, monocyte, lymphocyte, platelet, CD4, and CD8; the presence of lymphocytopenia and anemia; the levels of AST, ALT, total bilirubin, albumin, aPTT, PTT, serum creatinine, BUN, Hs-Troponin I, creatine kinase, 


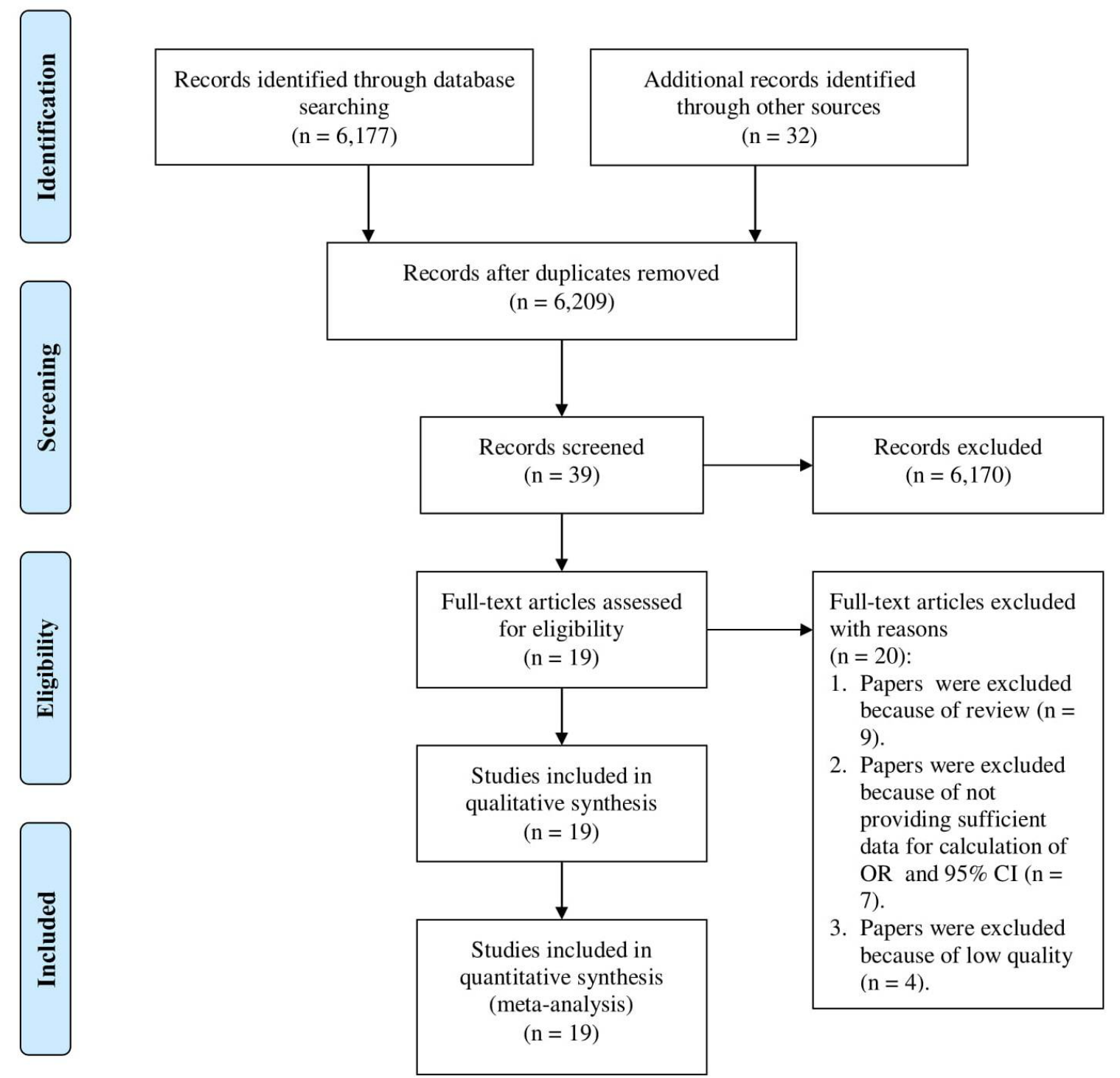

Figure 1. A flowchart of paper selection in our study.

IL-6, Hs-CRP, glucose, D-dimer, sodium, potassium, lactate dehydrogenase, and procalcitonin; elevated CRP; and ESR. Accordingly, the data were analyzed using the random effect model. The data for the remaining parameters were analyzed using the fixed effect model.

\section{Potential publication bias}

We used Egger's test to assess the potential publication bias. Our cumulative calculation revealed that reporting or publication bias $(\mathrm{p}<0.05)$ existed with respect to chronic liver disease, expectoration, myalgia, abdominal pain, heart rate, leukocytosis, elevated ESR, and elevated IL-6 levels.

\section{Discussion}

Our data suggest that comorbidities, such as chronic respiratory disease, cardiovascular disease, diabetes, and hypertension, were associated with a higher risk of severe COVID-19, among which, hypertension was the strongest risk factor. These results are consistent with those of previous meta-analyses ${ }^{43,44}$ that indicated that chronic respiratory disease, cardiovascular disease, diabetes, and hypertension are significantly associated with higher COVID-19 mortality. Hypertension and diabetes are also associated with higher mortality among patients with dengue fever, West Nile virus infection, Zika virus infection, and yellow fever ${ }^{45}$. To date, no study has reported details of the primary mechanism underlying the association between severe COVID-19 and comorbid factors. However, immune responses might be the most crucial factor underlying this association. Patients with comorbidities such as cardiovascular disease, chronic respiratory disease, hypertension, and diabetes were observed to have a lower immunity status than healthy individuals ${ }^{46-48}$. Since COVID-19 primarily affects the respiratory tract $^{49}$, 
Table 1. Baseline characteristics of studies included in our analysis.

\begin{tabular}{|c|c|c|c|c|c|c|c|}
\hline \multirow{2}{*}{ Author \& year } & \multirow{2}{*}{ Country } & \multirow{2}{*}{ City } & \multirow{2}{*}{ Hospital } & \multicolumn{2}{|c|}{ Sample size } & \multirow{2}{*}{ Outcome measure } & \multirow{2}{*}{ NOS } \\
\hline & & & & Severe & Mild & & \\
\hline Bai et al. $2020^{26}$ & China & Wuhan & Jinyintan Hospital & 91 & 36 & Died vs. cured & 7 \\
\hline Cai et al. $2020^{27}$ & China & Shenzen & Third people's Hospital & 58 & 240 & Severe vs. non severe & 9 \\
\hline Chen et al. $2020^{28}$ & China & Wuhan & Tongji hospital & 11 & 10 & Severe vs. moderate & 9 \\
\hline Chen et al. $2020^{29}$ & China & Mixed & Multicenter & 50 & 241 & Severe vs. mild-moderate & 9 \\
\hline Chen et al. $2020^{30}$ & China & Wuhan & Zhongnan Hospital & 14 & 11 & $\begin{array}{l}\text { Viral clearance vs. without } \\
\text { viral clearance }\end{array}$ & 9 \\
\hline Duan et al. $2020^{31}$ & China & Wuhan & Wuhan Pulmonary Hospital & 44 & 72 & Uncured vs. cured & 9 \\
\hline Gao et al. $2020^{32}$ & China & Fuyang & Second People's Hospital & 15 & 28 & Severe vs. mild & 7 \\
\hline Guan et al. $2020^{33}$ & China & Guangdong & $\begin{array}{l}\text { National Health Commision } \\
\text { of China }\end{array}$ & 926 & 173 & Severe vs. non-severe & 7 \\
\hline Huang et al. $2020^{34}$ & China & Wuhan & Jinyintan hospital & 13 & 28 & ICU vs. non-ICU & 9 \\
\hline Jian-Ya et al. $2020^{35}$ & China & Chongqing & Three Gorges Hospital & 7 & 44 & Severe vs. non severe & 9 \\
\hline Liu et al. $2020^{36}$ & China & Wuhan & Union Hospital & 69 & 69 & Severe vs. non severe & 7 \\
\hline Shi et al. $2020^{37}$ & China & Wuhan & Renmin Hospital & 48 & 53 & Died $<3$ d vs. $>3 d$ & 9 \\
\hline Wang et al. $2020^{38}$ & China & Mixed & Multicenter & 50 & 115 & CT imaging score $>11$ vs. $<11$ & 8 \\
\hline Wang et al. $2020^{39}$ & China & Wuhan & $\begin{array}{l}\text { Wuhan First People's } \\
\text { Hospital }\end{array}$ & 22 & 283 & Survivor vs. non-survivor & 8 \\
\hline Wang et al. $2020^{43}$ & China & Wuhan & Zhongnan Hospital & 36 & 102 & ICU vs. non-ICU & 9 \\
\hline Xu et al. $2020^{40}$ & China & Mixed & Multicenter & 25 & 44 & Severe vs. mild & 8 \\
\hline Zhang et al. $2020^{41}$ & China & Wuhan & Zhongnan Hospital & 55 & 166 & Severe vs. non-severe & 9 \\
\hline Zhang et al. $2020^{44}$ & China & Wuhan & Wuhan Seventh Hospital & 56 & 82 & Severe vs. non-severe & 7 \\
\hline Zhou et al. $2020^{42}$ & China & Wuhan & Wuhan Pulmonary Hospital & 54 & 137 & Survivor vs. non-survivor & 8 \\
\hline
\end{tabular}

Note: ICU, intensive care unit; CT, computed tomography; NOS, Newcastle Ottawa Scale.

\section{Table 2. Clinical characteristics of Covid-19 patients and the risk of severity.}

\begin{tabular}{|c|c|c|c|c|c|c|c|c|c|}
\hline \multirow{2}{*}{ Clinical characteristics } & \multirow{2}{*}{ NS } & \multirow{2}{*}{ Model } & \multicolumn{2}{|c|}{ Value } & \multirow{2}{*}{$\mathrm{pE}$} & \multirow{2}{*}{ pHet } & \multirow{2}{*}{$\mathbf{p}$} & \multirow{2}{*}{ OR } & \multirow{2}{*}{$95 \% \mathrm{CI}$} \\
\hline & & & Severe & Mild & & & & & \\
\hline \multicolumn{10}{|l|}{ Comorbids } \\
\hline Chronic kidney disease & 6 & Random & $14[3.94]$ & $15[1.68]$ & 1.3430 & 0.0280 & 0.1910 & 2.56 & $0.63-10.45$ \\
\hline Chronic liver disease & 6 & Fixed & $16[4.82]$ & $26[4.04]$ & $<0.0001$ & 0.3220 & 0.3220 & 1.45 & $0.70-3.01$ \\
\hline Chronic respiratory disease & 10 & Fixed & $31[5.47]$ & $31[1.66]$ & 0.7060 & 0.1020 & 0.0010 & 2.48 & $1.44-4.27$ \\
\hline Cerebrovascular accident & 5 & Random & $20[5.54]$ & 30 [2.09] & 0.9110 & 0.0380 & 0.1850 & 2.02 & $0.71-5.70$ \\
\hline Cardiovascular disease & 13 & Random & $76[10.45]$ & $94[4.95]$ & 0.5400 & 0.0580 & 0.0310 & 1.70 & $1.05-2.78$ \\
\hline Diabetes mellitus & 17 & Random & $156[19.24]$ & $194[8.40]$ & 0.7040 & $<0.0001$ & 0.0020 & 2.10 & $1.33-3.34$ \\
\hline Hypertension & 15 & Random & $269[35.54]$ & $369[16.79]$ & 0.7680 & $<0.0001$ & 0.0010 & 2.33 & $1.42-3.81$ \\
\hline Malignancy & 11 & Fixed & $29[4.43]$ & $40[2.23]$ & 0.6150 & 0.1430 & 0.5330 & 1.18 & $0.70-1.99$ \\
\hline
\end{tabular}




\begin{tabular}{|c|c|c|c|c|c|c|c|c|c|}
\hline \multirow{2}{*}{ Clinical characteristics } & \multirow{2}{*}{ NS } & \multirow{2}{*}{ Model } & \multicolumn{2}{|c|}{ Value } & \multirow{2}{*}{$\mathrm{pE}$} & \multirow{2}{*}{ pHet } & \multirow{2}{*}{$\mathbf{p}$} & \multirow{2}{*}{ OR } & \multirow{2}{*}{$95 \% \mathrm{CI}$} \\
\hline & & & Severe & Mild & & & & & \\
\hline \multicolumn{10}{|l|}{ Symptoms } \\
\hline Fever & 16 & Random & 599 [79.34] & 1932 [80.84] & 0.9220 & $<0.0001$ & 0.1730 & 1.51 & $0.83-2.74$ \\
\hline Cough & 12 & Random & 377 [64.33] & $1120[54.05]$ & 0.9560 & $<0.0001$ & 0.1890 & 1.53 & $0.81-2.90$ \\
\hline Dry cough & 4 & Fixed & 75 [44.38] & 178 [55.97] & 0.3130 & 0.1880 & 0.0360 & 0.66 & $0.44-0.97$ \\
\hline Expectoration & 10 & Fixed & 136 [26.67] & 438 [29.05] & $<0.0001$ & 0.8370 & 0.4970 & 1.09 & $0.85-1.39$ \\
\hline Sore throat & 10 & Random & 59 [10.57] & $196[10.96]$ & 0.7860 & 0.0040 & 0.6350 & 1.18 & $0.59-2.37$ \\
\hline Dyspnea & 13 & Random & $286[42.56]$ & 318 [16.51] & 0.6340 & $<0.0001$ & $<0.0001$ & 3.28 & $2.09-5.15$ \\
\hline Diarrhea & 13 & Random & 65 [9.62] & $134[6.68]$ & 0.5180 & 0.0690 & 0.8030 & 1.07 & $0.67-1.69$ \\
\hline Myalgia & 11 & Fixed & $105[17.89]$ & 283 [15.70] & $<0.0001$ & 0.7330 & 0.5160 & 1.10 & $0.831-1.44$ \\
\hline Nasal congestion & 4 & Fixed & $15[5.02]$ & $53[4.34]$ & 0.9350 & 0.1000 & 0.7590 & 1.12 & $0.55-2.29$ \\
\hline Anorexia & 9 & Random & 103 [25.37] & $143[15.10]$ & 0.6960 & 0.0040 & 0.0490 & 1.83 & $1.00-3.34$ \\
\hline Abdominal pain & 5 & Fixed & 15 [6.07] & $6[0.95]$ & $<0.0001$ & 0.5650 & 0.0040 & 3.91 & $1.53-10.02$ \\
\hline Fatigue & 13 & Random & 310 [46.48] & 694 [34.49] & 0.6790 & $<0.0001$ & 0.0040 & 2.00 & $1.25-3.20$ \\
\hline Dizziness & 4 & Fixed & $13[10.08]$ & $24[5.02]$ & 0.6510 & 0.1950 & 0.0180 & 2.67 & $1.18-6.01$ \\
\hline Headache & 11 & Fixed & $56[10.45]$ & $197[11.58]$ & 0.5070 & 0.1110 & 0.9950 & 1.00 & $0.71-1.41$ \\
\hline \multicolumn{10}{|l|}{ Signs } \\
\hline Temperature $>38^{\circ} \mathrm{C}$ & 5 & Random & 200 [57.97] & $738[50.14]$ & 0.6090 & 0.0020 & 0.2660 & 1.44 & $0.76-2.73$ \\
\hline Heart rate $(x / m i n)$ & 4 & Fixed & $269 \pm 35.54$ & $87.88 \pm 13.30$ & $<0.0001$ & 0.4070 & 0.0010 & 1.79 & $1.25-2.56$ \\
\hline Respiratory rate (x/min) & 5 & Random & $22.6 \pm 4.80$ & $20.36 \pm 2.00$ & 0.8080 & $<0.0001$ & 0.0100 & 2.85 & $1.28-6.33$ \\
\hline $\mathrm{SBP}(\mathrm{mmHg})$ & 5 & Fixed & $132.57 \pm 23.16$ & $123.88 \pm 14.37$ & 0.3340 & 0.1560 & $<0.0001$ & 1.84 & $1.31-2.60$ \\
\hline $\mathrm{DBP}(\mathrm{mmHg})$ & 3 & Random & $76.50 \pm 10.61$ & $75.59 \pm 9.89$ & 0.5350 & 0.0260 & 0.7190 & 1.14 & $0.56-2.32$ \\
\hline
\end{tabular}

Note, Value, data were presented in number [\%] or mean \pm SD; NS, number of studies; $p E, p$ Egger; pHet, p heterogeneity; OR, odd ratio; CI, confidence interval; SBP, systolic blood pressure; DBP, diastolic blood pressure.

\section{Table 3. Laboratory findings and the risk of severity in Covid-19 patients.}

\begin{tabular}{|c|c|c|c|c|c|c|c|c|c|}
\hline \multirow{2}{*}{$\begin{array}{c}\text { Clinical } \\
\text { characteristics }\end{array}$} & \multirow{2}{*}{ NS } & \multirow{2}{*}{ Model } & \multicolumn{2}{|c|}{ Value } & \multirow{2}{*}{ pE } & \multirow{2}{*}{ pHet } & \multirow{2}{*}{$\mathbf{p}$} & \multirow{2}{*}{ OR } & \multirow{2}{*}{$95 \% \mathrm{CI}$} \\
\hline & & & Severe & Mild & & & & & \\
\hline \multicolumn{10}{|c|}{ Complete Blood Count } \\
\hline WBC (10^9/L) & 14 & Random & $7.32 \pm 3.84$ & $5.17 \pm 2.04$ & 1.4980 & $<0.0001$ & $<0.0001$ & 4.92 & 2.12-11.31 \\
\hline Leukocytosis & 6 & Fixed & $62[26.00]$ & $40[6.03]$ & 0.0000 & 0.5940 & $<0.0001$ & 5.38 & $3.36-8.62$ \\
\hline Leukopenia & 6 & Fixed & 44 [18.00] & 206 [31.07] & 0.2890 & 0.2480 & 0.0160 & 0.59 & $0.41-0.87$ \\
\hline $\begin{array}{l}\text { Neutrophil count } \\
\left(10^{\wedge} 9 / \mathrm{L}\right)\end{array}$ & 12 & Random & $5.96 \pm 3.62$ & $3.84 \pm 2.12$ & 1.6380 & $<0.0001$ & 0.0010 & 5.45 & $2.04-14.54$ \\
\hline $\begin{array}{l}\text { Lymphocyte count } \\
\left(10^{\wedge} 9 / L\right)\end{array}$ & 15 & Random & $0.74 \pm 0.36$ & $1.03 \pm 0.44$ & 0.6440 & $<0.0001$ & $<0.0001$ & 0.34 & $0.23-0.50$ \\
\hline Lymphocytopenia & 6 & Random & $158[59.00]$ & 40 [6.03] & 0.8270 & $<0.0001$ & $<0.0001$ & 3.19 & $1.14-7.07$ \\
\hline
\end{tabular}




\begin{tabular}{|c|c|c|c|c|c|c|c|c|c|}
\hline \multirow{2}{*}{$\begin{array}{c}\text { Clinical } \\
\text { characteristics }\end{array}$} & \multirow{2}{*}{ NS } & \multirow{2}{*}{ Model } & \multicolumn{2}{|c|}{ Value } & \multirow{2}{*}{$\mathrm{pE}$} & \multirow{2}{*}{ pHet } & \multirow{2}{*}{ p } & \multirow{2}{*}{ OR } & \multirow{2}{*}{$95 \% \mathrm{CI}$} \\
\hline & & & Severe & Mild & & & & & \\
\hline \multicolumn{10}{|l|}{ Complete Blood Count } \\
\hline $\begin{array}{l}\text { Monocyte count } \\
\left(10^{\wedge} 9 / \mathrm{L}\right)\end{array}$ & 6 & Random & $0.38 \pm 0.17$ & $0.36 \pm 0.15$ & 0.5860 & 0.0100 & 0.5100 & 1.22 & $0.68-2.20$ \\
\hline Hemoglobin (g/L) & 9 & Fixed & $129.11 \pm 16.98$ & $132.02 \pm 17.50$ & 0.0900 & 0.4000 & 0.0460 & 0.76 & $0.58-1.00$ \\
\hline Anaemia & 2 & Random & $18[17.00]$ & 39 [10.32] & 0.7640 & 0.0660 & 0.4730 & 1.58 & $0.45-5.56$ \\
\hline Platelet count (10^9/L) & 12 & Random & $172.58 \pm 69.19$ & $183.21 \pm 62.50$ & 0.5550 & 0.0010 & 0.8200 & 0.82 & $0.55-1.23$ \\
\hline \multicolumn{10}{|l|}{ Physiological function } \\
\hline AST (U/L) & 11 & Random & $56.20 \pm 35.83$ & $28.67 \pm 11.18$ & 0.6930 & $<0.0001$ & $<0.0001$ & 4.91 & $2.96-8.12$ \\
\hline $\mathrm{ALT}(\mathrm{U} / \mathrm{L})$ & 12 & Random & $38.65 \pm 22.90$ & $25.60 \pm 14.71$ & 0.8060 & $<0.0001$ & $<0.0001$ & 3.23 & $1.90-5.52$ \\
\hline Total bilirubin $(\mu \mathrm{mol} / \mathrm{L})$ & 7 & Random & $15.80 \pm 9.50$ & $13.46 \pm 4.62$ & 1.6600 & $<0.0001$ & 0.5800 & 1.46 & $0.41-5.21$ \\
\hline Albumin ( $\mathrm{g} / \mathrm{L})$ & 6 & Random & $32.39 \pm 3.64$ & $35.53 \pm 3.71$ & 2.3900 & $<0.0001$ & 0.0950 & 0.19 & $0.03-1.34$ \\
\hline aPTT (s) & 7 & Random & $31.23 \pm 5.02$ & $33.13 \pm 3.66$ & 1.1900 & $<0.0001$ & 0.3420 & 0.58 & $0.19-1.79$ \\
\hline PTT (s) & 11 & Random & $13.45 \pm 1.86$ & $12.53 \pm 1.31$ & 0.7700 & $<0.0001$ & 0.2430 & 0.56 & $0.21-1.48$ \\
\hline $\begin{array}{l}\text { Serum creatinine } \\
(\mu \mathrm{mol} / \mathrm{L})\end{array}$ & 13 & Random & $82.04 \pm 31.69$ & $70.25 \pm 20.87$ & 0.6670 & $<0.0001$ & 0.0010 & 2.14 & $1.37-3.33$ \\
\hline BUN (mmol/L) & 10 & Random & $6.71 \pm 2.70$ & $4.74 \pm 1.38$ & 1.0220 & $<0.0001$ & $<0.0001$ & 6.15 & $3.05-12.37$ \\
\hline Hs-Troponin I (pg/ml) & 6 & Random & $31.9 \pm 61.55$ & $3.55 \pm 3.71$ & 1.1290 & $<0.0001$ & $<0.0001$ & 9.25 & $3.51-24.37$ \\
\hline Creatine kinase (U/L) & 10 & Random & $121.13 \pm 115.63$ & $77.47 \pm 56.26$ & 0.4860 & 0.0030 & $<0.0001$ & 2.44 & $1.65-3.62$ \\
\hline \multicolumn{10}{|l|}{ Inflammation markers } \\
\hline $\mathrm{Hs}-\mathrm{CRP}(\mathrm{mg} / \mathrm{L})$ & 10 & Random & $73.25 \pm 49.97$ & $29.96 \pm 24.40$ & 1.5600 & $<0.0001$ & $<0.0001$ & 14.27 & $5.13-39.71$ \\
\hline $\mathrm{CRP}>8 \mathrm{mg} / \mathrm{L}$ & 3 & Random & $147[83.10]$ & $254[52]$ & 1.1590 & 0.0050 & 0.0060 & 8.34 & $1.85-37.62$ \\
\hline $\operatorname{ESR}(\mathrm{mm} / \mathrm{h})$ & 4 & Random & $50.60 \pm 27.25$ & $29.19 \pm 26.52$ & 0.4200 & 0.0710 & $<0.0001$ & 4.45 & $2.56-7.76$ \\
\hline Elevated ESR & 2 & Fixed & 73 [68.00] & 214 [44.49] & $<0.0001$ & 0.8060 & $<0.0001$ & 2.80 & $1.78-4.39$ \\
\hline IL-6 (pg/ml) & 8 & Random & $30.45 \pm 31.29$ & $11.06 \pm 10.89$ & 0.9120 & $<0.0001$ & $<0.0001$ & 6.68 & $3.20-13.94$ \\
\hline Elevated IL-6 & 2 & Fixed & $44[66]$ & 115 [46.56] & $<0.0001$ & 0.7160 & 0.0200 & 1.98 & $1.12-3.52$ \\
\hline CD4 count(10^9/L) & 3 & Random & $217.19 \pm 118.56$ & $337.87 \pm 149.93$ & 1.5920 & 0.0010 & 0.2760 & 0.34 & $0.05-2.39$ \\
\hline CD8 count (10^9/L) & 3 & Random & $178.80 \pm 95.77$ & $224.17 \pm 76.36$ & 1.4260 & 0.0030 & 0.1420 & 0.26 & $0.04-1.57$ \\
\hline \multicolumn{10}{|l|}{ Others } \\
\hline Glucose (mmol/L) & 3 & Random & $7.04 \pm 1.83$ & $6.45 \pm 1.33$ & 0.9480 & 0.0030 & 0.3340 & 1.80 & $0.55-5.90$ \\
\hline D-dimer (pg/mL) & 15 & Random & $111.34 \pm 145.12$ & $38.88 \pm 28.93$ & 0.6070 & $<0.0001$ & $<0.0001$ & 6.19 & $4.22-9.08$ \\
\hline Serum Ferritin $(\mu \mathrm{g} / \mathrm{L})$ & 4 & Fixed & $1062.90 \pm 868.19$ & $600.67 \pm 758.61$ & 0.4310 & 0.1070 & 0.0310 & 1.96 & $1.06-3.62$ \\
\hline Sodium (mmol/L) & 3 & Random & $137.40 \pm 3.13$ & $92.39 \pm 1.77$ & 3.2770 & $<0.0001$ & 0.2840 & 11.93 & $0.13-1109.37$ \\
\hline Potassium (mmol/L) & 3 & Random & $4.12 \pm 0.61$ & $4.00 \pm 0.54$ & 0.9630 & 0.0010 & 0.7470 & 1.21 & $0.32-0.75$ \\
\hline $\begin{array}{l}\text { Lactate dehydrogenase } \\
(\mathrm{U} / \mathrm{L})\end{array}$ & 9 & Random & $381.85 \pm 159.44$ & $283.03 \pm 89.40$ & 0.6840 & $<0.0001$ & $<0.0001$ & 8.28 & $4.75-14.46$ \\
\hline Procalcitonin (ng/mL) & 10 & Random & $0.40 \pm 0.29$ & $0.12 \pm 0.07$ & 0.9880 & $<0.0001$ & $<0.0001$ & 6.62 & $3.32-13.21$ \\
\hline
\end{tabular}

Note: Value, data were presented in number [\%] or mean $\pm \mathrm{SD}$; NS, number of studies; $\mathrm{pE}$, p Egger; pHet, p heterogeneity; OR, odd ratio; CI, confidence interval; CBC, complete blood count; WBC, white blood cells; AST, aspartate transaminase; ALT, alanine transaminase; aPTT, activated partial thromboplastin time; PTT, partial thromboplastin time; BUN, blood urea nitrogen; Hs-CRP, high sensitivity C reactive protein; ESR, erythrocyte sedimentation rate; IL, interleukin. 
A).

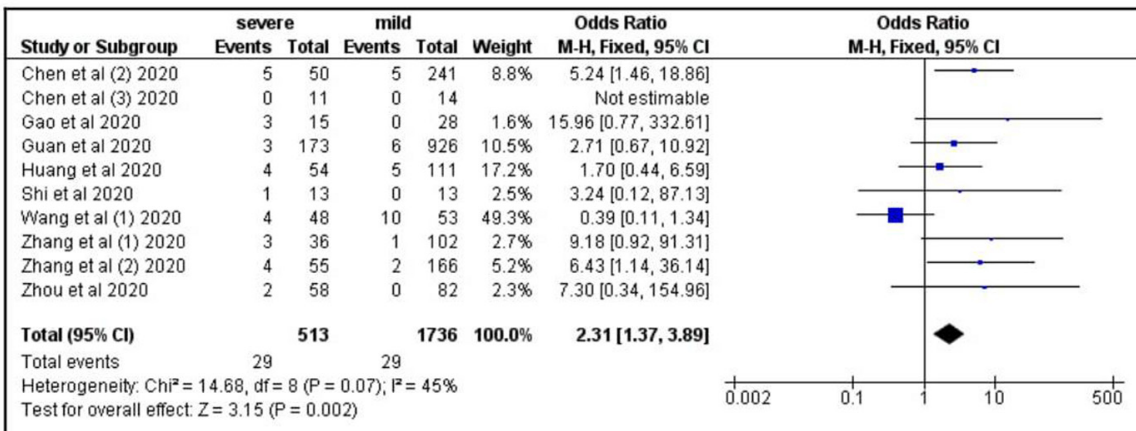

B).

\begin{tabular}{|c|c|c|c|c|c|c|c|c|c|}
\hline Study or Subgroup & \multicolumn{2}{|c|}{ severe } & \multicolumn{2}{|c|}{ mild } & Weight & $\begin{array}{c}\text { Odds Ratio } \\
\text { M-H, Random, } 95 \% \mathrm{Cl}\end{array}$ & \multicolumn{3}{|c|}{$\begin{array}{c}\text { Odds Ratio } \\
\text { M-H, Random, } 95 \% \mathrm{Cl}\end{array}$} \\
\hline Bai et al 2020 & 3 & 91 & 2 & 16 & $5.1 \%$ & $0.24[0.04,1.56]$ & & & - \\
\hline Chen et al (2) 2020 & 3 & 50 & 7 & 241 & $7.8 \%$ & $2.13[0.53,8.55]$ & & - & \\
\hline Chen et al (3) 2020 & 1 & 11 & 1 & 14 & $2.5 \%$ & $1.30[0.07,23.43]$ & & & \\
\hline Gao et al 2020 & 1 & 15 & 2 & 28 & $3.2 \%$ & $0.93[0.08,11.16]$ & & & \\
\hline Guan et al 2020 & 10 & 173 & 17 & 926 & $13.4 \%$ & $3.28[1.48,7.29]$ & & & \\
\hline Huang et al 2020 & 3 & 13 & 3 & 13 & $5.3 \%$ & $1.00[0.16,6.20]$ & & & \\
\hline Liu et al 2020 & 6 & 69 & 0 & 11 & $2.4 \%$ & $2.35[0.12,44.72]$ & & & \\
\hline Shi et al 2020 & 9 & 48 & 15 & 53 & $11.8 \%$ & $0.58[0.23,1.50]$ & & & - \\
\hline Wang et al (1) 2020 & 9 & 36 & 11 & 102 & $11.4 \%$ & $2.76[1.03,7.35]$ & & & \\
\hline Wang et al (2) 2020 & 12 & 54 & 22 & 111 & $13.5 \%$ & $1.16[0.52,2.56]$ & & & \\
\hline Zhang et al (1) 2020 & 13 & 55 & 9 & 166 & $12.1 \%$ & $5.40[2.16,13.49]$ & & & \\
\hline Zhang et al (2) 2020 & 4 & 58 & 3 & 82 & $6.8 \%$ & $1.95[0.42,9.07]$ & & & \\
\hline Zhou et al 2020 & 2 & 54 & 2 & 137 & $4.7 \%$ & $2.60[0.36,18.91]$ & & & \\
\hline Total $(95 \% \mathrm{Cl})$ & & 727 & & 1900 & $100.0 \%$ & $1.71[1.05,2.78]$ & & & \\
\hline Total events & 76 & & 94 & & & & & & \\
\hline $\begin{array}{l}\text { Heterogeneity: } \mathrm{Tau}^{2}= \\
\text { Test for overall effect: }\end{array}$ & $\begin{array}{l}0.29 ; \mathrm{Chi}^{2} \\
=2.15(\mathrm{~F}\end{array}$ & $\begin{array}{l}=20.6 \\
=0.03\end{array}$ & 1) $d f=12$ & $(P=0$ & $.06) ; 1^{2}=$ & & 0.01 & 1 & 10 \\
\hline
\end{tabular}

C).

\begin{tabular}{|c|c|c|c|c|c|c|c|c|c|c|}
\hline \multirow[b]{2}{*}{ Study or Subgroup } & \multicolumn{2}{|c|}{ severe } & \multicolumn{2}{|c|}{ mild } & \multirow[b]{2}{*}{ Weight } & \multicolumn{2}{|l|}{ Odds Ratio } & \multirow{2}{*}{\multicolumn{2}{|c|}{$\begin{array}{c}\text { Odds Ratio } \\
\text { M-H, Random, } 95 \% \mathrm{CI}\end{array}$}} & \\
\hline & Events & Total & Events & Total & & M-H, Random, $95 \% \mathrm{Cl}$ & & & & \\
\hline Bai et al 2020 & 10 & 91 & 5 & 16 & $6.2 \%$ & $0.27[0.08,0.94]$ & & & & \\
\hline Chen et al (1) 2020 & 2 & 11 & 1 & 10 & $2.5 \%$ & $2.00[0.15,26.19]$ & & & & \\
\hline Chen et al (2) 2020 & 7 & 50 & 15 & 241 & $7.6 \%$ & $2.45[0.94,6.37]$ & & & $\rightarrow$ & \\
\hline Chen et al (3) 2020 & 2 & 11 & 2 & 14 & $3.3 \%$ & $1.33[0.16,11.36]$ & & & & \\
\hline Duan et al 2020 & 16 & 44 & 11 & 72 & $7.9 \%$ & $3.17[1.30,7.71]$ & & & $\longrightarrow$ & \\
\hline Gao et al 2020 & 6 & 15 & 1 & 28 & $3.1 \%$ & $18.00[1.90,170.34]$ & & & & \\
\hline Guan et al 2020 & 28 & 173 & 53 & 926 & $9.9 \%$ & $3.18[1.95,5.19]$ & & & $\rightarrow$ & \\
\hline Huang et al 2020 & 1 & 13 & 7 & 13 & $2.9 \%$ & $0.07[0.01,0.72]$ & & & & \\
\hline Jian-Ya et al 2020 & 4 & 7 & 0 & 44 & $1.8 \%$ & $114.43[5.06,2585.61]$ & & & & \\
\hline Liu et al 2020 & 11 & 69 & 0 & 11 & $2.1 \%$ & $4.52[0.25,82.27]$ & & & & \\
\hline Shi et al 2020 & 9 & 48 & 13 & 53 & $7.5 \%$ & $0.71[0.27,1.85]$ & & & - & \\
\hline Wang et al (1) 2020 & 8 & 36 & 6 & 102 & $6.6 \%$ & $4.57[1.46,14.28]$ & & & & \\
\hline Wang et al (2) 2020 & 12 & 54 & 12 & 111 & $7.9 \%$ & $2.36[0.98,5.67]$ & & & $\rightarrow$ & \\
\hline Wang et al (3) 2020 & 6 & 22 & 25 & 283 & $7.2 \%$ & $3.87[1.39,10.78]$ & & & $\longrightarrow$ & \\
\hline Zhang et al (1) 2020 & 7 & 55 & 15 & 166 & $7.6 \%$ & $1.47[0.57,3.81]$ & & & — & \\
\hline Zhang et al (2) 2020 & 8 & 58 & 9 & 82 & $7.2 \%$ & $1.30[0.47,3.59]$ & & & - & \\
\hline Zhou et al 2020 & 19 & 54 & 19 & 137 & $8.7 \%$ & $3.37[1.61,7.06]$ & & & $\rightarrow$ & \\
\hline Total $(95 \%$ Cl) & & 811 & & 2309 & $100.0 \%$ & $2.10[1.32,3.34]$ & & & 4 & \\
\hline Total events & 156 & & 194 & & & & & & & \\
\hline $\begin{array}{l}\text { Heterogeneity: Tauz } \\
\text { Test for overall effect }\end{array}$ & $\begin{array}{l}0.50 ; \mathrm{Chi}^{2} \\
=3.15(\mathrm{~F}\end{array}$ & $\begin{array}{l}=43.2 \\
=0.00\end{array}$ & $\begin{array}{l}8, d f=16 \\
\text { 2) }\end{array}$ & $(P=0$ & $.0003) ; i^{2}$ & $=63 \%$ & 0.001 & 0.1 & 10 & 1000 \\
\hline
\end{tabular}

D).

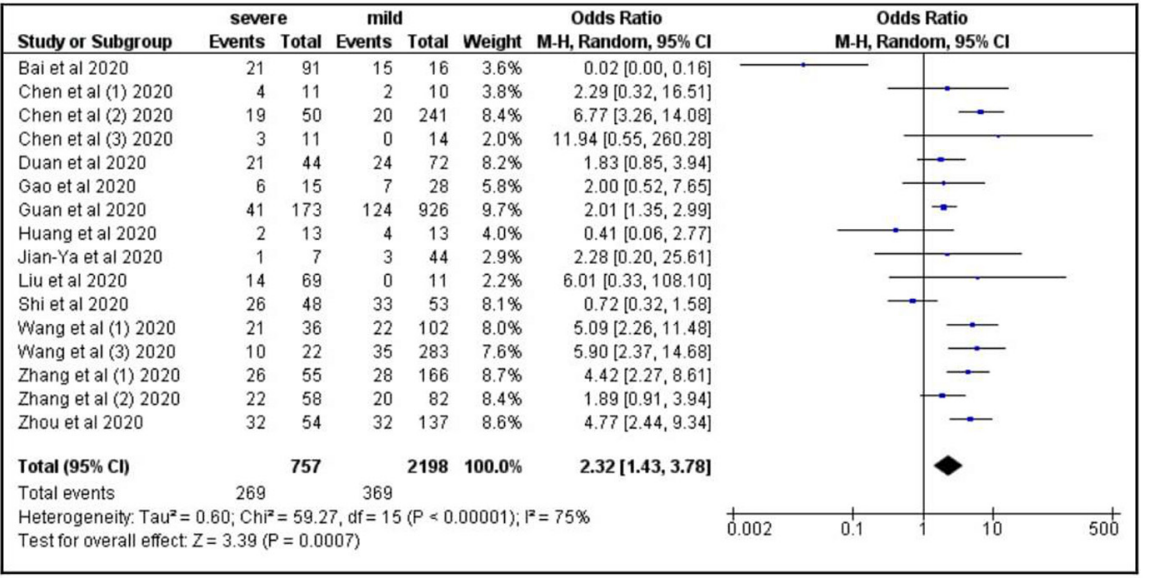

Figure 2. A forest plot of the association between comorbid factors and the risk of severe COVID-19. A) Chronic respiratory disease; B) Cardiovascular diease; C) Diabetes mellitus; D) Hypertension. 
A).

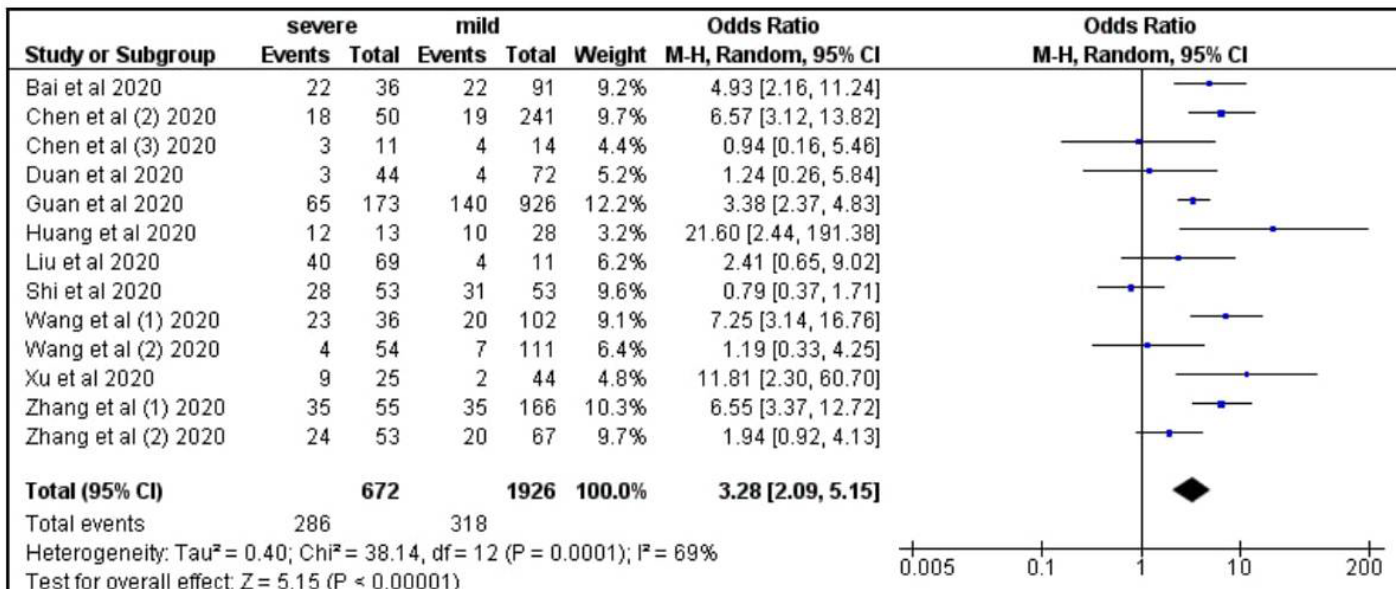

B).

Test for overall effect: $Z=5.15(P<0.00001)$

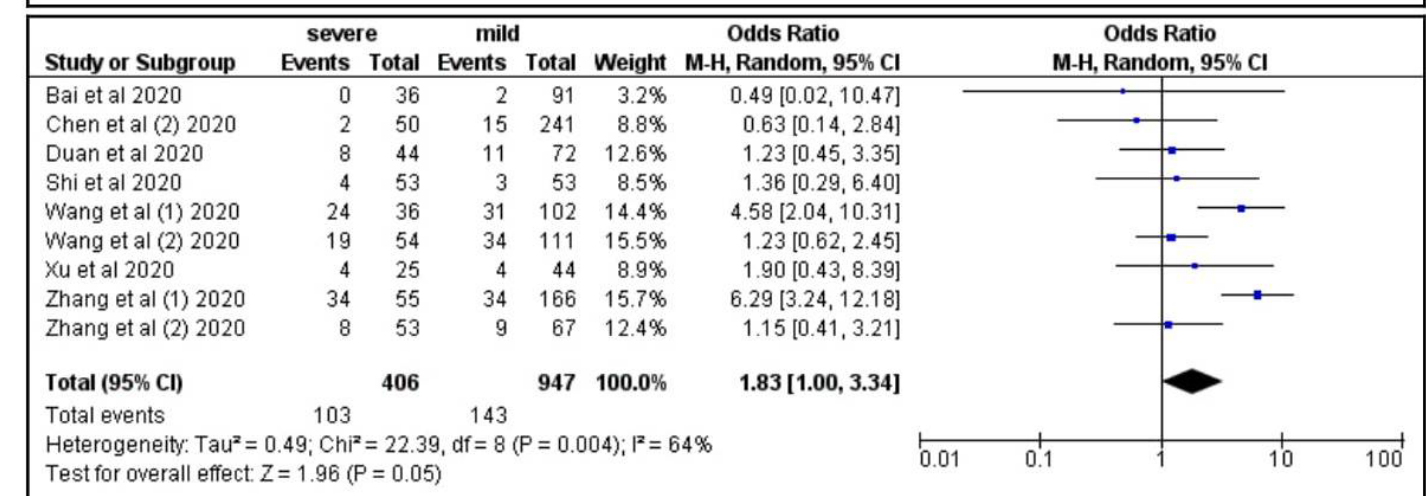

C).

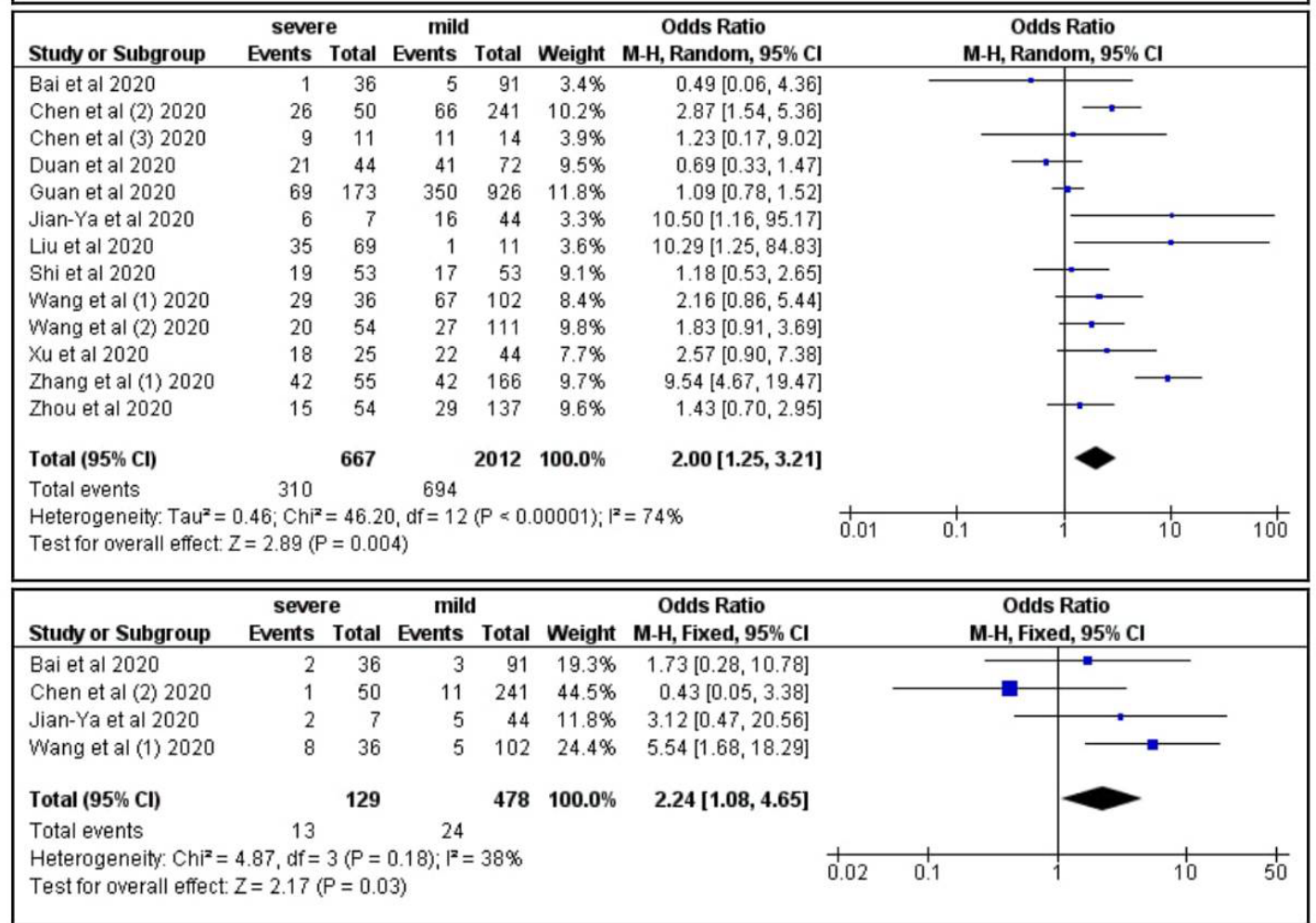

Figure 3. A forest plot of the association between clinical manifestations and the risk of severe COVID-19. A) Dyspnea; B) Anorexia; C) Fatique; D) Dizziness. 
A).

\begin{tabular}{|c|c|c|c|c|c|c|c|c|c|c|c|c|}
\hline \multirow[b]{2}{*}{ Study or Subgroup } & \multicolumn{3}{|c|}{ severe } & \multicolumn{2}{|c|}{ mild } & \multicolumn{3}{|c|}{ Std. Mean Difference } & \multirow{2}{*}{\multicolumn{4}{|c|}{$\begin{array}{c}\text { Std. Mean Difference } \\
\text { IV, Random, } 95 \% \mathrm{Cl}\end{array}$}} \\
\hline & Mean & SD & Total & Mean & SD T & Total $n$ & Weight & IV, Random, 95\% Cl & & & & \\
\hline Bai et al 2020 & 26 & 6.18 & 36 & 22 & 0.75 & 91 & $20.8 \%$ & $1.19[0.78,1.61]$ & & & $\longrightarrow$ & \\
\hline Chen et al (2) 2020 & 20.67 & 1.53 & 50 & 19.45 & 1.46 & 241 & $22.6 \%$ & $0.83[0.51,1.14]$ & & & $\longrightarrow-$ & \\
\hline Chen et al (3) 2020 & 20.33 & 2.54 & 11 & 20.33 & 2.47 & 14 & $13.9 \%$ & $0.00[-0.79,0.79]$ & & & & \\
\hline Duan et al 2020 & 19 & 1.53 & 44 & 19 & 1.51 & 72 & $21.5 \%$ & $0.00[-0.38,0.38]$ & & & & \\
\hline Shi et al 2020 & 27 & 12.23 & 53 & 21 & 3.81 & 53 & $21.2 \%$ & $0.66[0.27,1.05]$ & & & & \\
\hline Total $(95 \% \mathrm{Cl})$ & & & 194 & & & 4711 & $100.0 \%$ & $0.57[0.14,1.01]$ & & & & \\
\hline \multicolumn{8}{|c|}{$\begin{array}{l}\text { Heterogeneity. } \text { Tau }^{2}=0.20 ; \mathrm{Chi}^{2}=21.95, \mathrm{df}=4(\mathrm{P}=0.0002) ; 1^{2}=82 \% \\
\text { Test for overall effect: } Z=2.57(P=0.01)\end{array}$} & & -2 & -1 & 0 & 2 \\
\hline & \multicolumn{3}{|c|}{ severe } & \multicolumn{3}{|c|}{ mild } & \multicolumn{3}{|c|}{ Std. Mean Difference } & \multirow{2}{*}{\multicolumn{2}{|c|}{$\begin{array}{l}\text { Std. Mean Difference } \\
\text { IV, Fixed, } 95 \% \mathrm{Cl}\end{array}$}} & \\
\hline Study or Subgroup & Mean & SD & Total & Mean & SD & Total & Weight & IV, Fixed, $95 \% \mathrm{Cl}$ & & & & \\
\hline Bai et al 2020 & 134.67 & 16.21 & 36 & 126.67 & 14.31 & 91 & $23.3 \%$ & $0.53[0.14,0.93]$ & & & $\longrightarrow$ & \\
\hline Chen et al (2) 2020 & 124 & 15.27 & 50 & 122.28 & 14.27 & 241 & $38.5 \%$ & $0.12[-0.19,0.42]$ & & & & \\
\hline Chen et al (3) 2020 & 134.2 & 27.1 & 11 & 126.8 & 22.6 & 14 & $5.7 \%$ & $0.29[-0.50,1.08]$ & & & & \\
\hline Duan et al 2020 & 125 & 20.69 & 44 & 120.33 & 12.1 & 72 & $25.2 \%$ & $0.29[-0.09,0.67]$ & & & $\because-$ & \\
\hline Huang et al 2020 & 145 & 36.54 & 13 & 123.33 & 8.59 & 28 & $7.4 \%$ & $0.99[0.29,1.68]$ & & & & \\
\hline Total $(95 \% \mathrm{Cl})$ & & & 154 & & & 446 & $100.0 \%$ & $0.33[0.14,0.52]$ & & & & \\
\hline \multicolumn{6}{|c|}{$\begin{array}{l}\text { Heterogeneity: } \mathrm{Chi}^{2}=6.39, \mathrm{df}=4(P=0.17) ; \mathrm{I}^{2}=37 \% \\
\text { Test for overall effect: } Z=3.46(P=0.0005)\end{array}$} & & & & -2 & -1 & 0 & 2 \\
\hline
\end{tabular}

Figure 4. A forest plot of the association between clinical manifestation and the risk of severe COVID-19. A) Respiratory rate; B) Systolic blood pressure.

patients with chronic respiratory diseases might be at a higher risk of contracting severe COVID-19. In addition, endothelial dysfunction might also play a pivotal role ${ }^{50}$.

COVID-19 is a novel disease, and the immune response of this disease is not completely understood. Our data suggest that elevated leukocyte and neutrophil levels and reduced lymphocyte levels are associated with severe COVID-19. In other viral infections, such as influenza, elevated leukocyte and neutrophil levels serve as important predictors of disease severity ${ }^{51}$. The role of leukocytes in the pathogenesis of COVID-19 is conflicting. In most cases, viral infections have been observed to cause leukopenia ${ }^{52}$. Furthermore, a study also reported that leukopenia was observed at a significantly higher frequency among

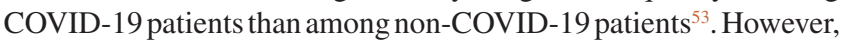
in our present study, we did not compare COVID-19 and non-COVID-19 patients. The major factor that seemed to affect our findings was the occurrence of cytokine storm in patients. In COVID-19, there is an immune system overreaction, which results in a cytokine storm. In this condition, leukocytes might be over-activated, which might lead to the release of high levels of cytokines ${ }^{54}$. Consistent with our data, a study has confirmed that cytokine storm is significantly associated with severe COVID-195. The theory underlying the role of neutrophils in COVID-19, as reported in our study, remains unclear. The speculations might be attributed to the involvement of neutrophil extracellular traps (NETs). While no study has assessed the precise role of NETs in COVID-19 pathogenesis, certain researchers speculate that SARS-CoV-2 might stimulate neutrophils to produce NETs, similar to several other viral pathogens ${ }^{56}$. Furthermore, this might lead to neutrophil infiltration in pulmonary capillaries, organ damage, and the development of acute respiratory distress syndrome ${ }^{57}$.
Low lymphocyte levels were observed in patients with severe COVID-19 compared with those with mild COVID-19. In the context of the immunological mechanism, our results might be contradictory. Lymphocyte subsets are known to play an important role in the action against bacterial, viral, fungal, and parasitic infections ${ }^{58}$; therefore, the levels of circulating lymphocytes should increase. The immunological response in COVID-19 is unique and remains unclear. However, certain propositions might help describe our findings. First, coronaviruses infect human cells through ACE2 receptors ${ }^{59}$. Since ACE2 receptors are also expressed by lymphocytes ${ }^{60}$, the coronaviruses may enter lymphocytes and induce apoptosis. Second, the feedback mechanism between pro-inflammatory cytokines (such as IL-6) and lymphocytes might also explain our results. A study revealed that elevation in the levels of pro-inflammatory cytokines correlated with reduction in the levels of lymphocytes $^{61}$. Moreover, our findings also confirmed the significant elevation in the levels of IL-6. Third, ACE2 receptors are expressed by cells from various organs, including the thymus and spleen ${ }^{62}$. As coronaviruses infect human cells through the ACE2 receptors, the spleen and thymus might also be damaged in patients with COVID-19, which would lead to lower levels of lymphocyte production. Fourth, lymphocyte proliferation requires a balanced metabolism, and metabolic disorders such as hyperlactic acidemia have been reported to disturb lymphocyte proliferation ${ }^{63}$. Hyperlactic acidemia has been observed in patients with severe COVID-1964.

The studies included in this systematic review also suggest that the levels of D-dimer were significantly higher in patients with severe COVID-19. Coagulation in patients with COVID-19 has been a major concern, and the lack of reliable data and meta-analyses prevents a holistic comparison. Certain 
A).

\begin{tabular}{|c|c|c|c|c|c|c|c|c|c|c|c|c|}
\hline \multirow[b]{2}{*}{ Study or Subgroup } & \multicolumn{3}{|c|}{ severe } & \multicolumn{2}{|c|}{ mild } & \multirow[b]{2}{*}{ Total } & \multirow{2}{*}{ Weight } & \multicolumn{2}{|l|}{ Std. Mean Difference } & \multirow{2}{*}{\multicolumn{2}{|c|}{$\begin{array}{c}\text { Std. Mean Difference } \\
\text { IV, Random, 95\% Cl }\end{array}$}} & \\
\hline & Mean & SD & Total & Mean & SD & & & N, Random, $95 \% \mathrm{Cl}$ & & & & \\
\hline Chen et al (1) 2020 & 9.2 & 3.6 & 11 & 4.7 & 1.5 & 10 & $6.4 \%$ & $1.54[0.54,2.54]$ & & & - & \\
\hline Chen et al (2) 2020 & 4.62 & 2.06 & 50 & 4.67 & 1.32 & 241 & $8.4 \%$ & $-0.03[-0.34,0.27]$ & & & & \\
\hline Chen et al (3) 2020 & 4.5 & 2.2 & 11 & 4.5 & 1.7 & 14 & $7.1 \%$ & $0.00[-0.79,0.79]$ & & & - & \\
\hline Duan et al 2020 & 8.9 & 6.44 & 44 & 6.43 & 3.56 & 72 & $8.2 \%$ & $0.51[0.12,0.89]$ & & & - & \\
\hline Huang et al 2020 & 4.26 & 1.64 & 15 & 4.96 & 1.85 & 28 & $7.6 \%$ & $-0.39[-1.02,0.25]$ & & & & \\
\hline Jian-Ya et al 2020 & 8.1 & 4.99 & 22 & 4.87 & 2.31 & 283 & $8.1 \%$ & $1.25[0.80,1.69]$ & & & - & \\
\hline Shi et al 2020 & 6.63 & 6.61 & 7 & 5.63 & 2.68 & 44 & $7.0 \%$ & $0.29[-0.51,1.09]$ & & & & \\
\hline Wang et al (1) 2020 & 12.36 & 1.25 & 48 & 7.72 & 0.51 & 53 & $7.1 \%$ & $4.91[4.12,5.71]$ & & & $\rightarrow$ & \\
\hline Wang et al (3) 2020 & 9.73 & 5.23 & 13 & 5.47 & 3.52 & 28 & $7.4 \%$ & $1.01[0.32,1.71]$ & & & $\rightarrow$ & \\
\hline Xu et al 2020 & 6.67 & 4.79 & 36 & 4.33 & 1.58 & 102 & $8.2 \%$ & $0.84[0.44,1.23]$ & & & - & \\
\hline Zhang et al (1) 2020 & 4.6 & 1.73 & 25 & 4.47 & 1.69 & 44 & $8.0 \%$ & $0.08[-0.42,0.57]$ & & & - & \\
\hline Zhang et al (2) 2020 & 6.57 & 4.03 & 55 & 4.33 & 2.02 & 166 & $8.4 \%$ & $0.84[0.52,1.15]$ & & & - & \\
\hline Zhou et al 2020 & 6.1 & 3.8 & 56 & 4.63 & 1.81 & 82 & $8.3 \%$ & $0.52[0.18,0.87]$ & & & - & \\
\hline Total $(95 \% \mathrm{Cl})$ & & & 393 & & & 1167 & $100.0 \%$ & $0.84[0.35,1.33]$ & & & $>$ & \\
\hline $\begin{array}{l}\text { Heterogeneity: } \mathrm{Tau}^{2}= \\
\text { Test for overall effect: }\end{array}$ & $\begin{array}{l}0.72 ; \mathrm{Chi} \\
=3.36\end{array}$ & $\begin{array}{l}I^{2}=16 \\
(P=0 .\end{array}$ & $\begin{array}{l}3.20, d f \\
0008)\end{array}$ & $f=12(\mathrm{P}$ & $0<0.0$ & 0001); & $F^{2}=93 \%$ & & $\begin{array}{c}+10 \\
-10\end{array}$ & -5 & 5 & $\frac{1}{10}$ \\
\hline
\end{tabular}

B).

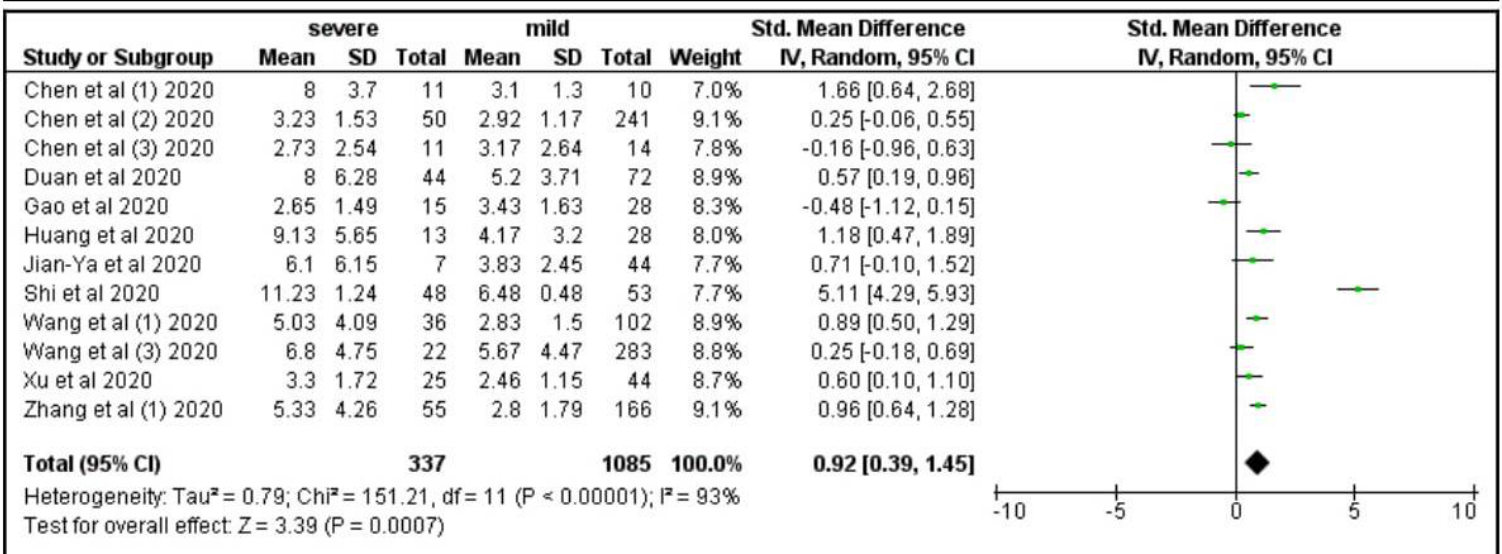

C).

\begin{tabular}{|c|c|c|c|c|c|c|c|c|c|c|}
\hline \multirow[b]{2}{*}{ Study or Subgroup } & \multicolumn{2}{|c|}{ severe } & \multicolumn{2}{|c|}{ mild } & \multirow[b]{2}{*}{ Weight } & \multicolumn{2}{|l|}{ Odds Ratio } & \multirow{2}{*}{\multicolumn{2}{|c|}{$\begin{array}{c}\text { Odds Ratio } \\
\text { M-H, Random, } 95 \% \mathrm{CI}\end{array}$}} & \\
\hline & Events & Total & Events & Total & & M-H, Random, $95 \% \mathrm{Cl}$ & & & & \\
\hline Cai et al 2020 & 39 & 58 & 75 & 240 & $21.1 \%$ & $4.52[2.45,8.33]$ & & & $\varpi$ & \\
\hline Chen et al (1) 2020 & 8 & 11 & 1 & 10 & $7.3 \%$ & $24.00[2.06,279.62]$ & & & & \\
\hline Chen et al (2) 2020 & 24 & 50 & 42 & 241 & $20.8 \%$ & $4.37[2.29,8.35]$ & & & $\rightarrow-$ & \\
\hline Huang et al 2020 & 11 & 13 & 15 & 28 & $11.6 \%$ & $4.77[0.89,25.57]$ & & & & \\
\hline Zhang et al (1) 2020 & 18 & 55 & 21 & 166 & $20.1 \%$ & $3.36[1.63,6.94]$ & & & $\rightarrow$ & \\
\hline Zhang et al (2) 2020 & 58 & 82 & 46 & 56 & $19.1 \%$ & $0.53[0.23,1.21]$ & & & & \\
\hline Total $(95 \% \mathrm{Cl})$ & & 269 & & 741 & $100.0 \%$ & $3.19[1.44,7.09]$ & & & & \\
\hline Total events & 158 & & 200 & & & & & & & \\
\hline $\begin{array}{l}\text { Heterogeneity: } \mathrm{Tau}^{2}= \\
\text { Test for overall effect: }\end{array}$ & $\begin{array}{l}69 ; \mathrm{Chi}^{2} \\
=2.85(\mathrm{H}\end{array}$ & $\begin{array}{l}=23.0 \\
=0.0\end{array}$ & $4, d f=5($ & $(P=0.0$ & $0003) ; 1^{2}=$ & $78 \%$ & 0.002 & 0.1 & 10 & 500 \\
\hline
\end{tabular}

D).

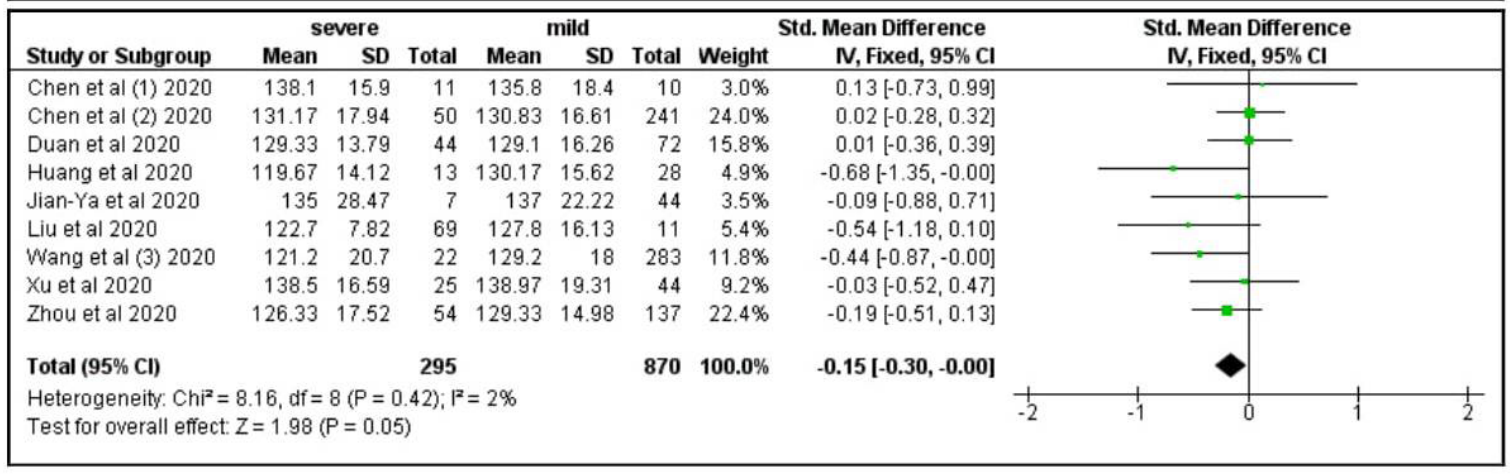

Figure 5. A forest of the association between complete blood count and the risk of severe COVID-19. A) White blood cells; B) Neutrophil count; C) Lymphocytopenia; D) Hemoglobin. 
A).

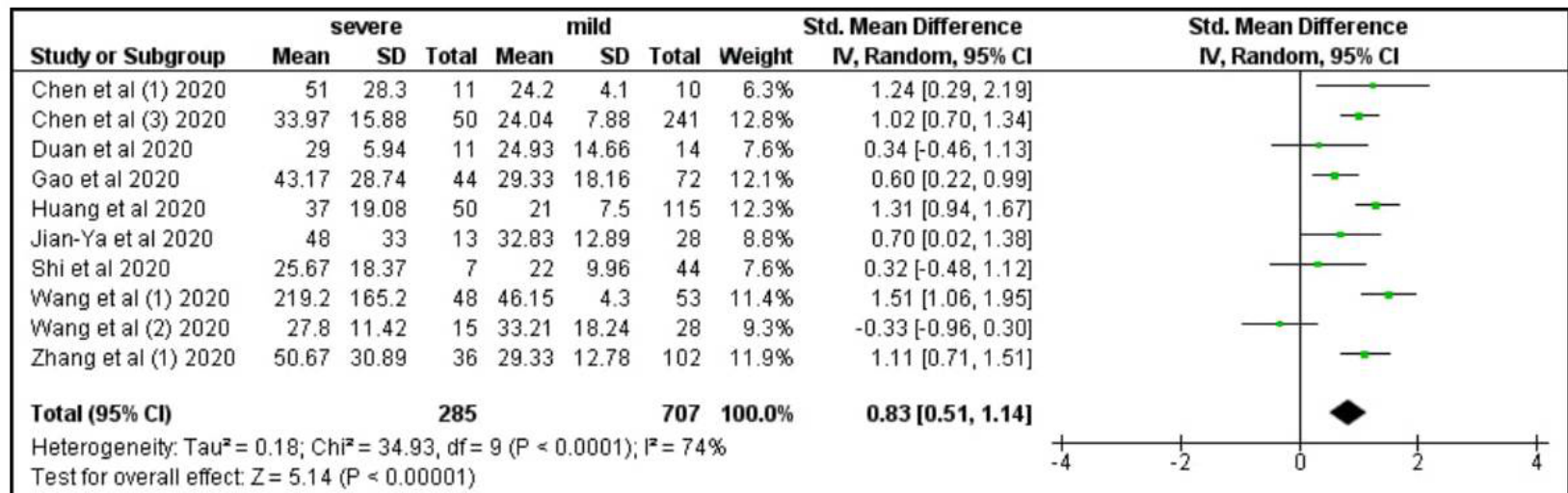

B).

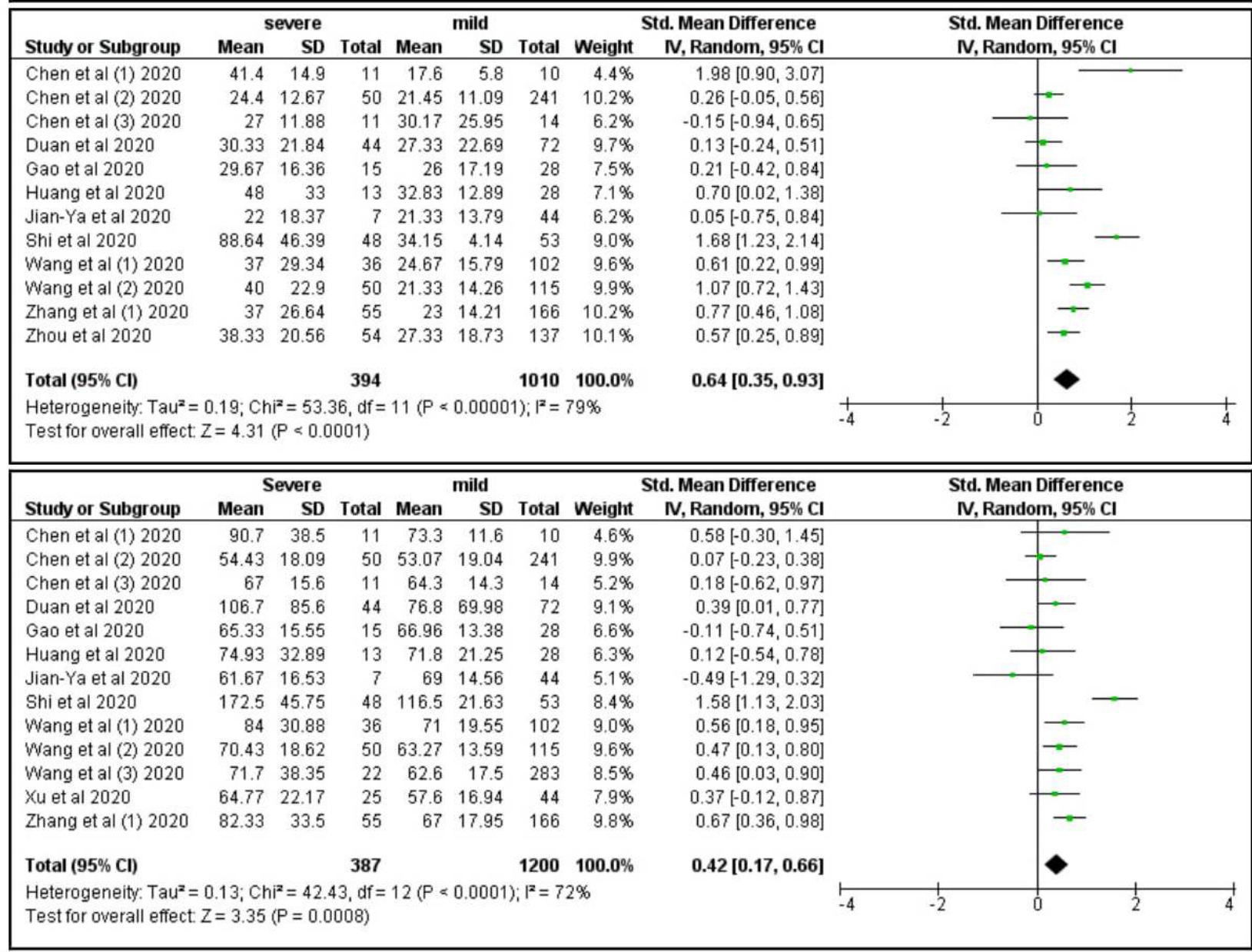

Figure 6. A forest plot of the association between the risk of severe COVID-19 and the levels of AST (A), ALT (B), and serum creatinine (C).

infectious diseases that cause abnormal coagulation have been associated with poor clinical outcomes ${ }^{65}$. The theory behind this mechanism is not understood clearly. It is widely known that ACE2 receptors are important for the infection of host cells by SARS-CoV-2, and ACE2 receptors are expressed in various cells in the human body, including endothelial cells ${ }^{66}$.
Consequently, a massive inflammatory reaction may occur in endothelial cells owing to SARS-CoV-2 infection ${ }^{67}$, which may lead to increased coagulation, disseminated intravascular coagulation $^{68}$, and increased fibrin degradation ${ }^{69}$. High fibrin degradation leads to elevated levels of fibrinogen and D-dimer ${ }^{70}$, which might also explain the occurrence of venous thromboembolism 
A).

\begin{tabular}{|c|c|c|c|c|c|c|c|c|c|c|}
\hline \multirow[b]{2}{*}{ Study or Subgroup } & \multicolumn{3}{|c|}{ severe } & \multicolumn{2}{|c|}{ mild } & \multirow[b]{2}{*}{ Total } & \multicolumn{2}{|r|}{ Std. Mean Difference } & \multirow{2}{*}{\multicolumn{2}{|c|}{$\begin{array}{c}\text { Std. Mean Difference } \\
\text { N, Random, } 95 \% \mathrm{Cl}\end{array}$}} \\
\hline & Mean & SD & Total & Mean & SD & & Weight & IN, Random, $95 \% \mathrm{Cl}$ & & \\
\hline Chen et al (1) 2020 & 7.7 & 4.37 & 11 & 4.2 & 1.3 & 10 & $7.2 \%$ & $1.02[0.10,1.94]$ & & \\
\hline Chen et al (2) 2020 & 4.56 & 1.79 & 50 & 3.83 & 1.02 & 241 & $11.4 \%$ & $0.61[0.31,0.92]$ & & $\rightarrow$ \\
\hline Duan et al 2020 & 7.63 & 2.07 & 44 & 5.23 & 1.97 & 72 & $10.8 \%$ & $1.19[0.78,1.59]$ & & \\
\hline Gao et al 2020 & 4.51 & 1.76 & 15 & 4.09 & 1.29 & 28 & $9.2 \%$ & $0.28[-0.35,0.91]$ & & \\
\hline Jian-Ya et al 2020 & 4.97 & 2.57 & 7 & 4.27 & 1.92 & 44 & $8.0 \%$ & $0.34[-0.46,1.14]$ & & \\
\hline Shi et al 2020 & 15.2 & 2.37 & 48 & 10.08 & 1.01 & 53 & $9.7 \%$ & $2.84[2.28,3.40]$ & & \\
\hline Wang et al (1) 2020 & 6.6 & 4.09 & 36 & 4.07 & 1.5 & 102 & $10.8 \%$ & $1.03[0.63,1.43]$ & & $\rightarrow$ \\
\hline Wang et al (2) 2020 & 4.1 & 1.67 & 50 & 3.5 & 1.13 & 115 & $11.2 \%$ & $0.45[0.12,0.79]$ & & $\rightarrow$ \\
\hline Wang et al (3) 2020 & 5.63 & 3.09 & 22 & 4 & 1.4 & 283 & $10.5 \%$ & $1.03[0.59,1.47]$ & & $\longrightarrow$ \\
\hline Zhang et al (1) 2020 & 6.2 & 3.2 & 55 & 4.1 & 1.29 & 166 & $11.3 \%$ & $1.08[0.76,1.40]$ & & $\rightarrow$ \\
\hline Total $(95 \% \mathrm{Cl})$ & & & 338 & & & 1114 & $100.0 \%$ & $0.99[0.61,1.37]$ & & \\
\hline $\begin{array}{l}\text { Heterogeneity: } \mathrm{Tau}^{2}= \\
\text { Test for overall effect: }\end{array}$ & $\begin{array}{l}.31 ; \mathrm{Ch} \\
=5.09\end{array}$ & $\begin{array}{l}P^{2}=65 \\
(P<0 .\end{array}$ & $\begin{array}{l}59, \mathrm{df}= \\
00001)\end{array}$ & $=9(P<$ & 0.0000 & $01) ; 1^{2}=$ & $=86 \%$ & & -4 & 0 \\
\hline
\end{tabular}

B).

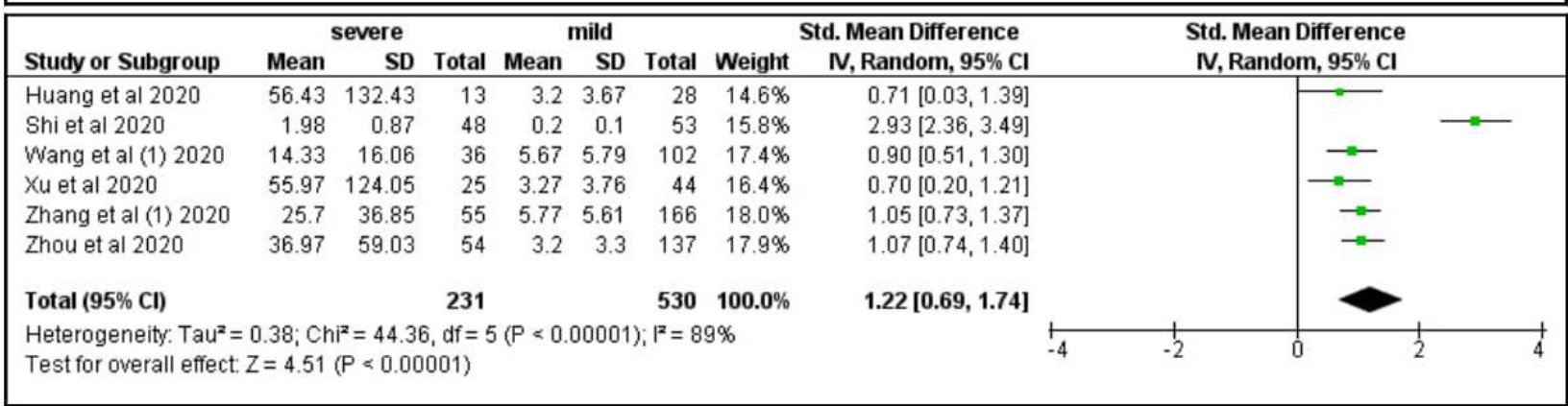

C).

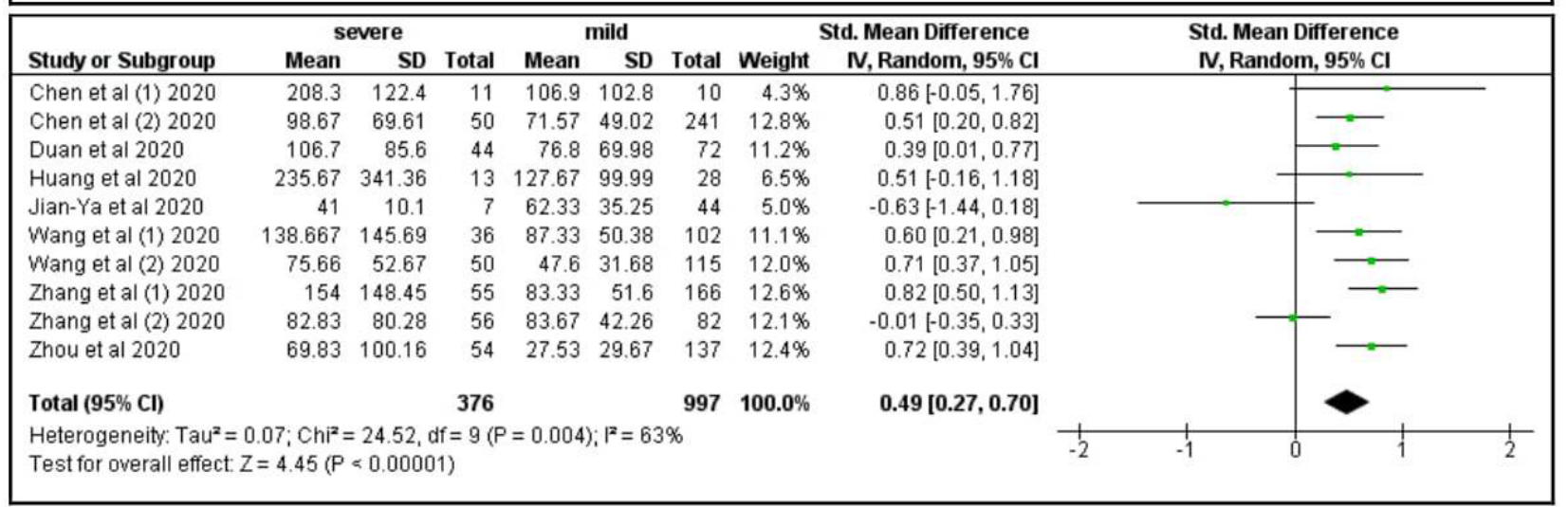

Figure 7. A forest plot of the association between the risk of severe COVID-19 and the levels of BUN (A), Hs-troponin (B), and creatine kinase $(\mathbf{C})$.

in critical patients of COVID-1971. In addition, a study with a short follow-up period also reported the existence of a dynamic correlation between the D-dimer levels and the severity of COVID-19 ${ }^{72}$. Furthermore, pulmonary embolism and deep vein thrombosis were also observed in patients with severe COVID-1973,74, which suggests that D-dimer might play a prominent role in governing the severity of COVID-19 patients.

We also observed that inflammatory markers, including elevated levels of CRP, ESR, and IL-6, were found both in patients with severe and mild COVID-19, with a significant increase detected in patients with severe COVID-19. Other variables associated with adverse outcomes, such as ferritin, lactate dehydrogenase, and procalcitonin levels, were found to be elevated predominantly in patients with severe COVID-19. Our findings were consistent with those of a previous meta-analysis ${ }^{75}$, and indicated that high levels of CRP, lactate dehydrogenase, and ESR were associated with adverse outcomes in COVID-19. Another meta-analysis had also confirmed that elevated levels of IL-6 were observed in patients with COVID-19 who exhibited poor clinical outcomes ${ }^{76}$. Therefore, the levels of CRP, ESR, IL-6, ferritin, procalcitonin, and lactate dehydrogenase can serve as potential markers for the evaluation of COVID-19 prognosis.

The high mortality rate and treatment failure in patients with COVID-19 can be attributed to the fact that COVID-19 affects 
A).

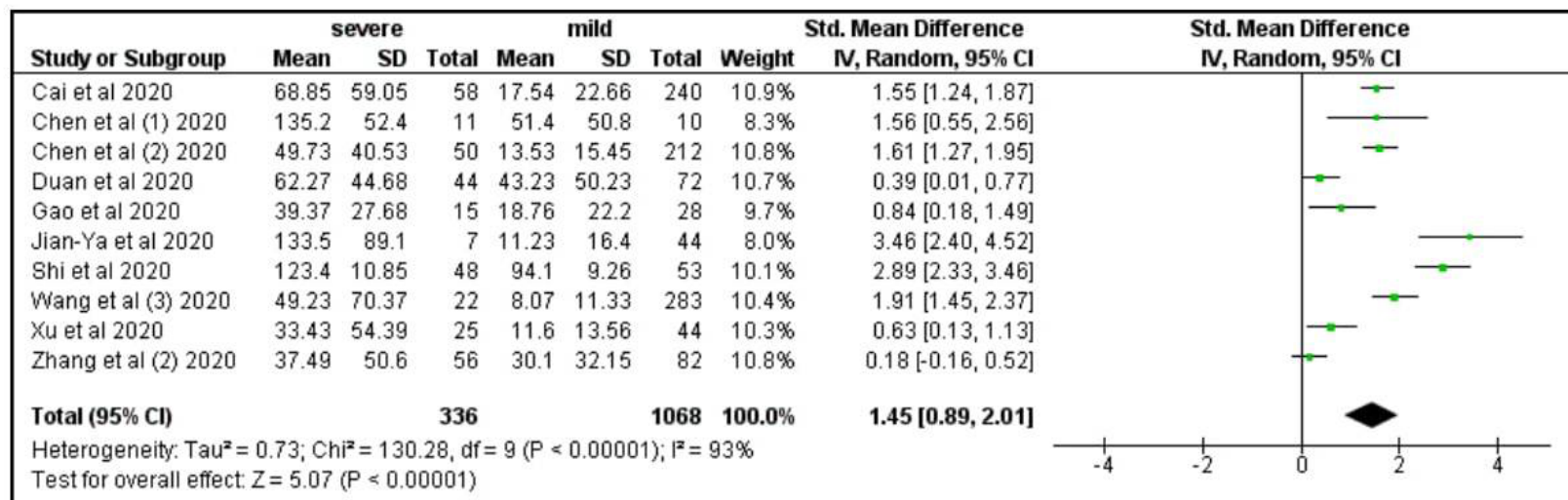

B).

\begin{tabular}{|c|c|c|c|c|c|c|c|c|c|}
\hline Study or Subgroup & \multicolumn{2}{|c|}{ severe } & \multicolumn{2}{|c|}{ mild } & Weight & $\begin{array}{c}\text { Odds Ratio } \\
\text { M-H, Random, } 95 \% \mathrm{Cl}\end{array}$ & \multicolumn{3}{|c|}{$\begin{array}{c}\text { Odds Ratio } \\
\text { M-H, Random, } 95 \% \mathrm{Cl}\end{array}$} \\
\hline Cai et al 2020 & 54 & 58 & 142 & 240 & $36.2 \%$ & $9.32[3.27,26.56]$ & & & $\longrightarrow$ \\
\hline Chen et al (2) 2020 & 34 & 50 & 111 & 241 & $40.5 \%$ & $2.49[1.30,4.75]$ & & & $\rightarrow-$ \\
\hline Liu et al 2020 & 59 & 69 & 1 & 11 & $23.3 \%$ & $59.00[6.79,512.69]$ & & & \\
\hline Total $(95 \% \mathrm{Cl})$ & & 177 & & 492 & $100.0 \%$ & $8.40[1.82,38.76]$ & & & \\
\hline Total events & 147 & & 254 & & & & & & \\
\hline $\begin{array}{l}\text { Heterogeneity: } \mathrm{Tau}^{2}= \\
\text { Test for overall effect }\end{array}$ & $\begin{array}{l}1.40 ; \mathrm{Ch} \\
\mathrm{Z}=2.73\end{array}$ & $\begin{array}{l}z=10.8 \\
P=0.0\end{array}$ & $\begin{array}{l}\text { 81, } d f=2 \\
06)\end{array}$ & $(P=0$. & $005) ; 1^{2}=$ & $81 \%$ & 0.002 & 0.1 & 10 \\
\hline
\end{tabular}

C).

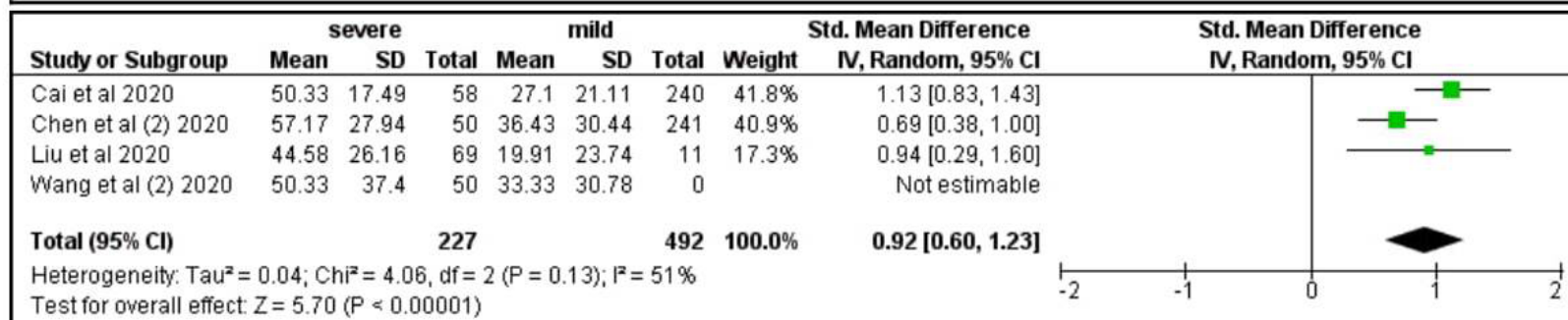

D).

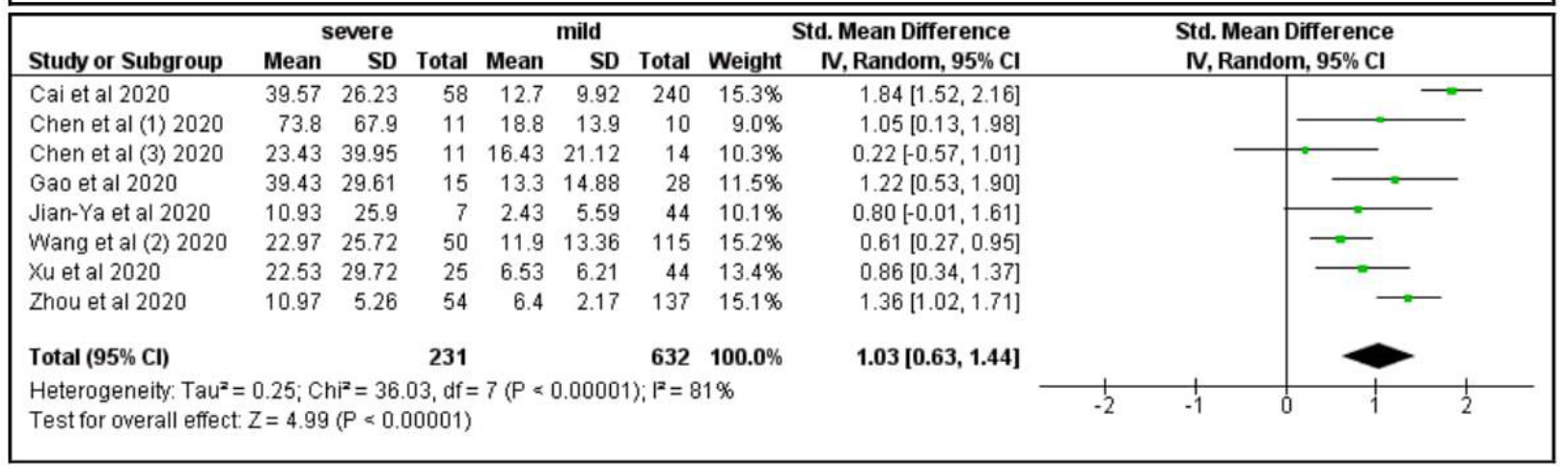

Figure 8. A forest plot of the association between the risk of severe COVID-19 and the levels of CRP (A), Hs-CRP (B), ESR (C), and IL-6 (D).

multiple organs, including the lung, heart, kidney, and $\operatorname{liver}^{77}$. Our data suggest that elevated levels of urea and creatinine, and not chronic kidney disease, were associated with severe COVID-19, which indicates that acute inflammation might be caused by SARS-CoV-2 infection. Previous meta-analyses have also reported findings consistent with our results ${ }^{78,79}$. Moreover, anatomical studies have reported significant renal inflammation in patients with severe COVID-1975,80,81. There might be two mechanisms by which SARS-CoV-2 induces renal inflammation. First, SARS-CoV-2 might directly infect renal tubular epithelial cells and podocytes through ACE2 receptors, which facilitates the targeted infection of certain cells by the virus. 
A).

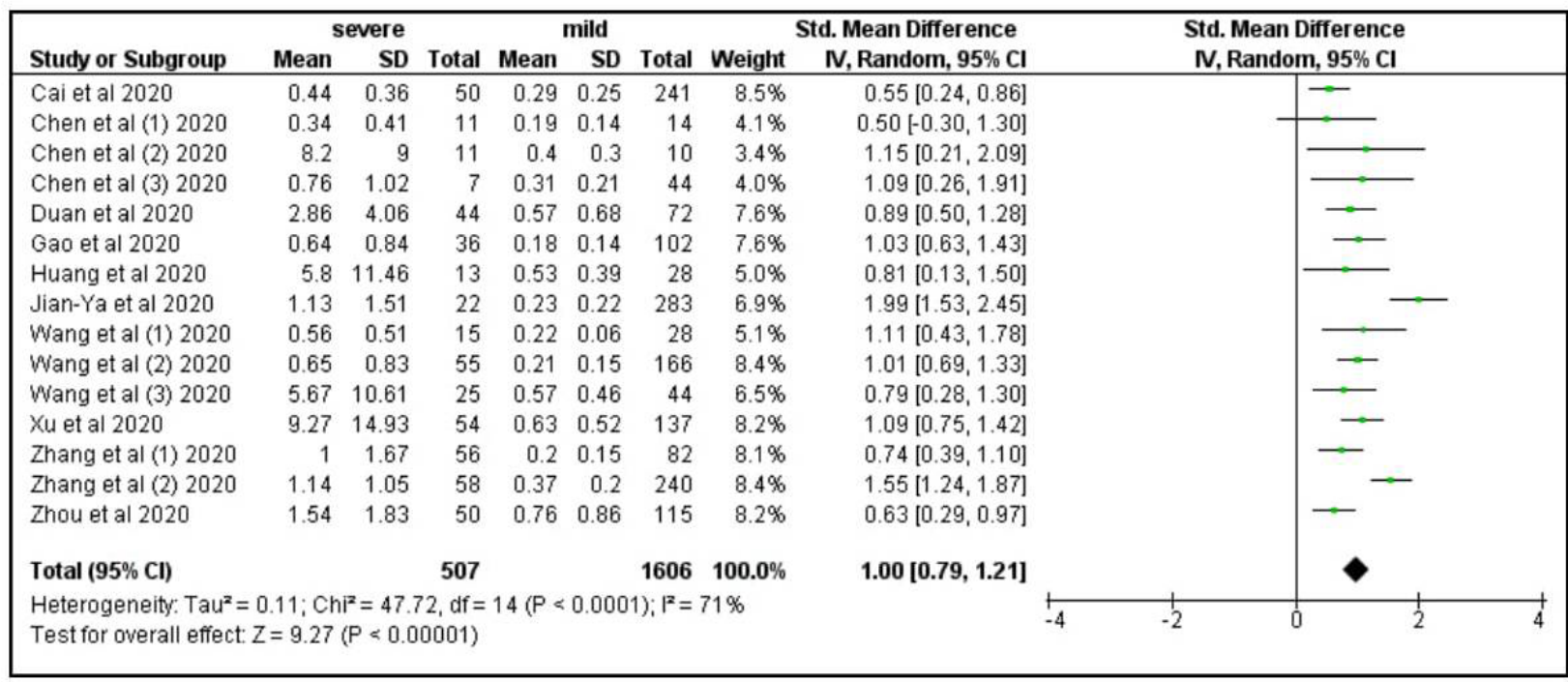

B).

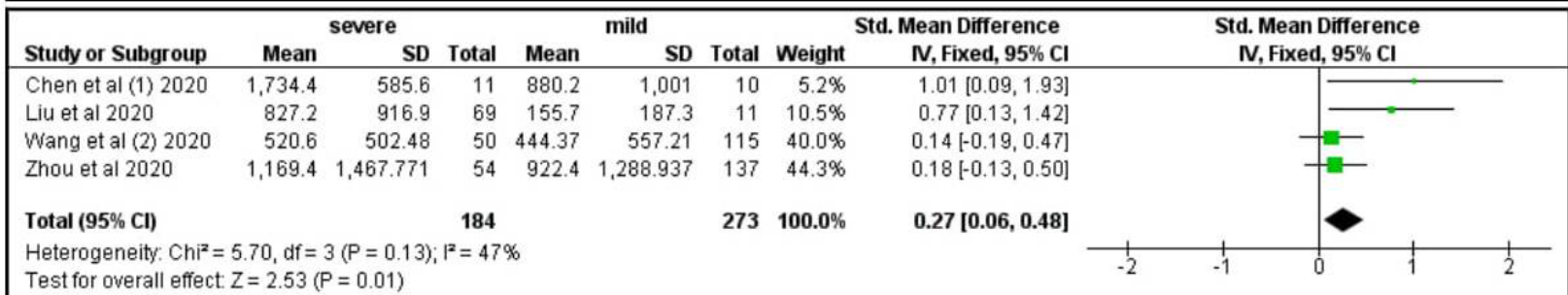

C).

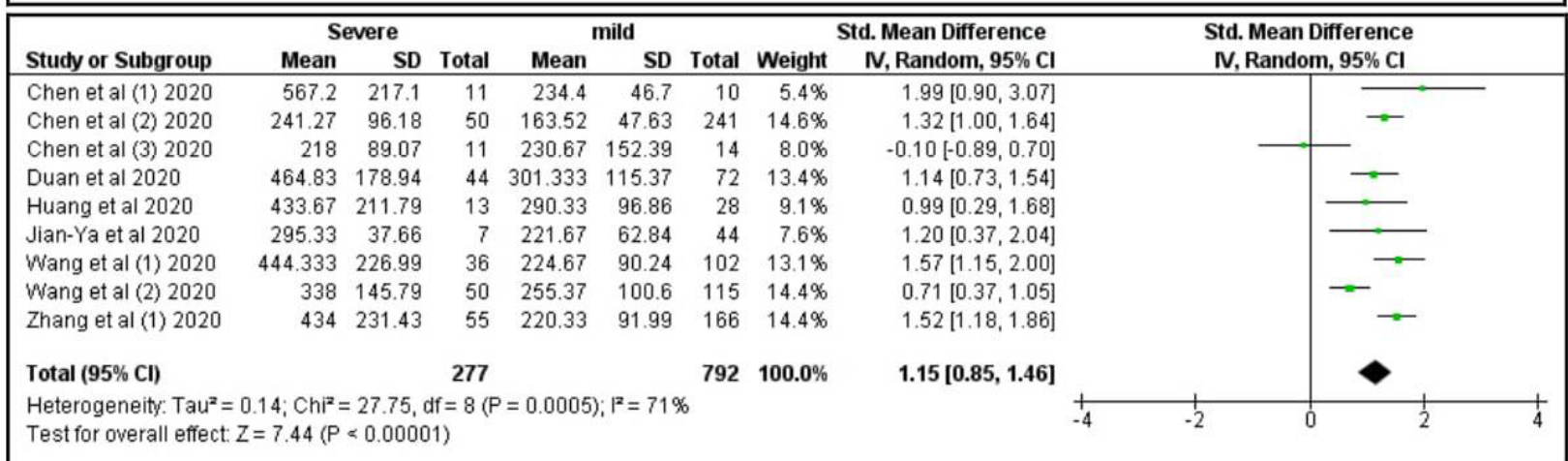

D).

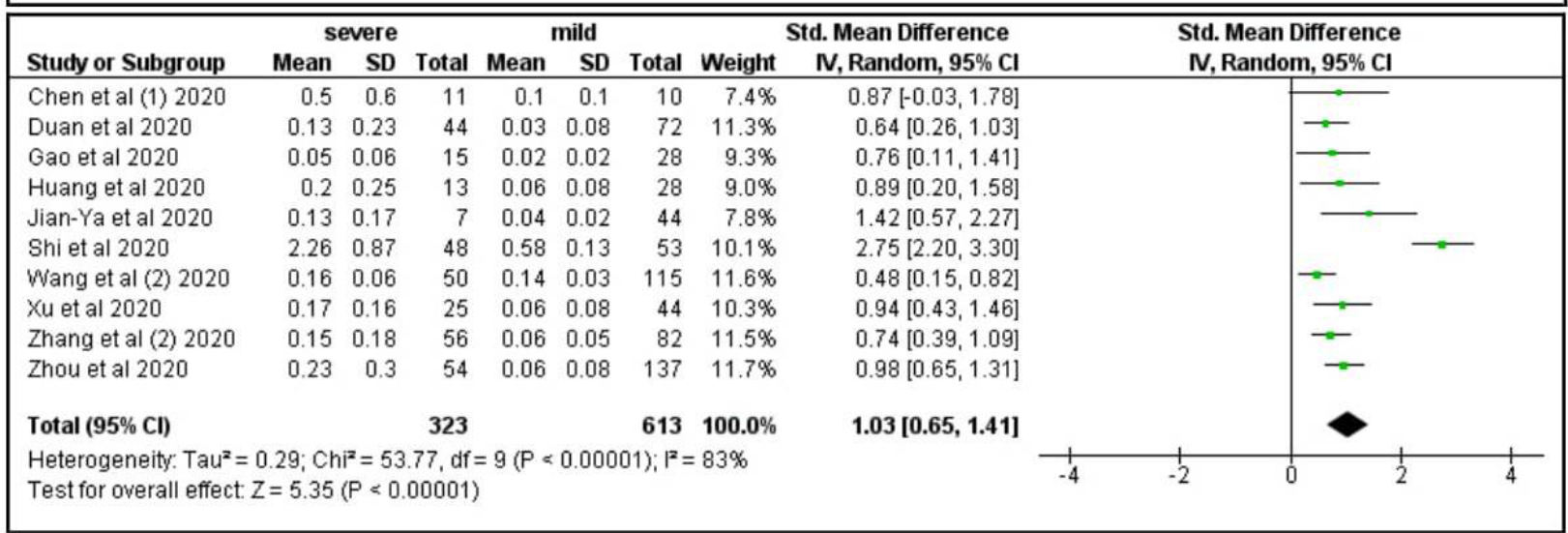

Figure 9. A forest plot of the association between the risk of severe COVID-19 and the levels of D-dimer (A), serum ferritin (B), lactate dehydrogenase $(\mathbf{C})$, and procalcitonin (D). 
Consequently, acute tubular necrosis, podocytopathy, microangiopathy, and collapsing glomerulopathy might occur owing to the massive inflammation in renal tubular epithelial cells and podocytes ${ }^{82,83}$. Second, the binding between SARS-CoV-2 and ACE2 receptors might activate angiotensin II and induce cytokine production, which may lead to hypercoagulopathy and microangiopathy, and eventually cause renal hypoxia ${ }^{84,85}$.

Conversely, with respect to liver function, we observed that the levels of liver enzymes were higher in patients with severe COVID-19. Previous studies in this context have elucidated that ACE2 receptors are highly expressed in bile duct cells; therefore, infection of these cells by coronaviruses might lead to abnormalities in the levels of liver enzymes ${ }^{86}$. However, a recent anatomical study on liver biopsy specimens from patients with severe COVID-19 revealed that moderate microvascular steatosis and mild lobular and portal activities were observed ${ }^{87}$. These data suggest that it cannot be determined clearly whether the elevated levels of liver enzymes in patients with severe COVID-19 are caused by direct infection or by drug-induced liver injury. Therefore, further studies are required to elucidate the precise mechanism underlying the elevation of liver enzymes levels in patients with severe COVID-19.

Meta-analyses on this topic have been performed previously $^{43,44,75,76,88-91}$. However, compared to previous studies, our study has the following strengths. The previous studies only reported limited factors, such as clinical manifestations ${ }^{43,88,90,91}$, laboratory findings ${ }^{76,89}$, or a combination of only clinical manifestations and laboratory findings ${ }^{75}$. In our study, we included all comorbidities, clinical manifestations, and laboratory characteristics. Additionally, compared to previous studies, this study has a larger sample size; the data on 1,934 patients with mild and 1,644 patients with severe COVID-19 treated across 19 hospitals were retrieved. However, this study also has certain limitations. Certain crucial factors that might play an important role in the pathogenesis of COVID-19, including secondary infection, treatment, and immunological status were not controlled for. Our current findings should be interpreted with caution because the majority of studies included were cross-sectional, and the samples corresponding to the data analyzed originated only in China. Longitudinal studies may reveal more long-term impacts of SARS-CoV-2 infection ${ }^{92}$.

\section{Conclusion}

COVID-19 is an emergent infectious disease, and the major problem associated with it is the unknown pattern of disease development. We identified 34 factors that are associated with severe COVID-19. This might improve our understanding of COVID19 progression and provide baseline data to compile or improve the prediction models for the estimation of COVID-19 prognosis.

\section{Data availability}

\section{Underlying data}

All data underlying the results are available as part of the article and no additional source data are required.

\section{Reporting guidelines}

Figshare: PRISMA checklist for 'Predictors of COVID-19 severity: a systematic review and meta-analysis', https://doi.org/ 10.6084/m9.figshare.12813683.v1 $1^{93}$

Data are available under the terms of the Creative Commons Attribution 4.0 International license (CC-BY 4.0).
1. Acikgoz O, Gunay A: The early impact of the Covid-19 pandemic on the global and Turkish economy. Turk J Med Sci. 2020; 50(SI-1): 520-526. PubMed Abstract | Publisher Full Text | Free Full Text

2. Nicola M, Alsafi Z, Sohrabi C, et al.: The socio-economic implications of the coronavirus pandemic (COVID-19): A review. Int J Surg. 2020; 78: 185-193. PubMed Abstract | Publisher Full Text | Free Full Text

3. Al-Tawfiq JA, Leonardi R, Fasoli G, et al.: Prevalence and fatality rates of COVID-19: What are the reasons for the wide variations worldwide? Travel Med Infect Dis. 2020; 35: 101711.

PubMed Abstract | Publisher Full Text | Free Full Text

4. Shojaee S, Pourhoseingholi MA, Ashtari S, et al.: Predicting the mortality due to Covid-19 by the next month for Italy, Iran and South Korea; a simulation study. Gastroenterol Hepatol Bed Bench. 2020; 13(2): 177-179. PubMed Abstract | Free Full Text

5. Wang L, Li J, Guo S, et al.: Real-time estimation and prediction of mortality caused by COVID-19 with patient information based algorithm. Sci Total Environ. 2020; 727: 138394

PubMed Abstract | Publisher Full Text | Free Full Text

6. Siordia JA: Epidemiology and clinical features of COVID-19: A review of current literature. J Clin Virol. 2020; 127: 104357.

PubMed Abstract | Publisher Full Text | Free Full Text

7. Bai $X$, Fang $C$, Zhou $Y$, et al.: Predicting COVID-19 malignant progression with AI techniques. 2020.

Publisher Full Text
8. Xie J, Hungerford $D$, Chen $H$, et al.: Development and external validation of a prognostic multivariable model on admission for hospitalized patients with COVID-19. Korean J Radiol. 2020; 21(8): 1007-1017. PubMed Abstract | Publisher Full Text | Free Full Text

9. Caramelo F, Ferreira N, Oliveiros B: Estimation of risk factors for COVID-19 mortality-preliminary results. MedRxiv. 2020. Publisher Full Text

10. Qi X, Jiang Z, Yu Q, et al:: Machine learning-based CT radiomics model for predicting hospital stay in patients with pneumonia associated with SARS-CoV-2 infection: A multicenter study. Ann Trans/ Med. 2020; 8(14): 859. PubMed Abstract | Publisher Full Text | Free Full Text

11. Yan $\mathrm{L}$, Zhang $\mathrm{HT}$, Xiao $\mathrm{Y}$, et al.: Prediction of criticality in patients with severe Covid-19 infection using three clinical features:a machine learning-based prognostic model with clinical data in Wuhan. MedRxiv. 2020. Publisher Full Text

12. Yuan $\mathrm{M}$, Yin $\mathrm{W}$, Tao Z, et al.: Association of radiologic findings with mortality of patients infected with 2019 novel coronavirus in Wuhan, China. PLOS One. 2020; 15(3): e0230548 PubMed Abstract | Publisher Full Text | Free Full Text

13. Lu J, Hu S, Fan R, et al.: ACP risk grade: a simple mortality index for patients with confirmed or suspected severe acute respiratory syndrome coronavirus 2 disease (COVID-19) during the early stage of outbreak in Wuhan, China. MedRxiv. 2020 Publisher Full Text 
14. Gong J, Ou J, Qiu X, et al.: A tool to early predict severe 2019-novel coronavirus pneumonia (COVID-19):a multicenter study using the risk nomogram in Wuhan and Guangdong, China. Clin Infect Dis. 2020; 71(15): 833-840.

PubMed Abstract | Publisher Full Text | Free Full Text

15. Shi Y, Yu X, Zhao H, et al.: Host susceptibility to severe COVID-19 and establishment of a host risk score: findings of $\mathbf{4 8 7}$ cases outside Wuhan. Crit Care. 2020; 24(1): 108

PubMed Abstract | Publisher Full Text | Free Full Text

16. Wynants L, Van Calster B, Collins GS, et al.: Prediction models for diagnosis and prognosis of covid-19 infection: systematic review and critical appraisal. BMJ. 2020; 369: $\mathrm{m} 1328$.

PubMed Abstract | Publisher Full Text | Free Full Text

17. Fajar J, Mahdi B, Heriansyah T, et al.: Length of stay and major adverse cardiac events: Comparison between percutaneous coronary intervention and thrombolytic therapy in patients with ST-elevation myocardial infarction Implications for cost effectiveness. Archives of Hellenic Medicine/ Arheia Ellenikes Iatrikes. 2019; 36(4). Reference Source

18. Fajar JK, Andalas M, Harapan H: Comparison of Apgar scores in breech presentations between vaginal and cesarean delivery. Tzu-Chi Medical Journal. 2017; 29(1): 24-29.

PubMed Abstract | Publisher Full Text | Free Full Text

19. Fajar JK, Harapan $\mathrm{H}$ : Socioeconomic and attitudinal variables associated with acceptance and willingness to pay towards dengue vaccine :a systematic review. Arch Clin Infect Dis. 2017; 12(3): e13914. Publisher Full Text

20. Fajar JK, Mahendra AI, Tamara F, et al: The association between complete blood count and the risk of coronary heart disease. Turkiye Klinikleri J Med Sci. 2019; 39(1): 56-64. Publisher Full Text

21. Fajar JK, Taufan T, Syarif M, et al.: Hip geometry and femoral neck fractures: A meta-analysis. J Orthop Translat 2018; 13:1-6. PubMed Abstract | Publisher Full Text | Free Full Text

22. Prihatiningsih S, Fajar JK, Tamara F, et al.: Risk factors of tuberculosis infection among health care workers: a meta-analysis. Indian J Tuberc. 2020; 67(1): 121-129.

PubMed Abstract | Publisher Full Text

23. Rohman MS, Purnamasari $Y$, Ilmawan $M$, et al.: Comparison of major bleeding in patients with acute coronary syndrome that underwent coronary artery bypass grafting treated with clopidogrel or ticagrelor: a systematic review and meta-analysis [version 1; peer review: 1 approved, 1 approved with reservations]. F1000Res. 2020; 9(99): 99. Publisher Full Text

24. McInnes MDF, Moher $\mathrm{D}$, Thombs $\mathrm{BD}$, et al.: Preferred Reporting Items for a Systematic Review and Meta-analysis of Diagnostic Test Accuracy Studies: The PRISMA-DTA Statement. JAMA. 2018; 319(4): 388-396. PubMed Abstract | Publisher Full Text

25. Stang A: Critical evaluation of the Newcastle-Ottawa scale for the assessment of the quality of nonrandomized studies in meta-analyses. Eur J Epidemiol. 2010; 25(9): 603-5. PubMed Abstract | Publisher Full Text

26. Bai T, Tu S, Wei $Y$, et al.: Clinical and laboratory factors predicting the prognosis of patients with COVID-19: an analysis of 127 patients in Wuhan, China. China (2/26/2020). 2020.

Publisher Full Text

27. Cai $Q$, Huang $D$, Ou P, et al.: COVID-19 in a designated infectious diseases hospital outside Hubei Province, China. Allergy. 2020; 75(7): 1742-1752. PubMed Abstract | Publisher Full Text

28. Chen G, Wu D, Guo W, et al.: Clinical and immunological features of severe and moderate coronavirus disease 2019. J Clin Invest. 2020; 130(5): 2620-2629.

PubMed Abstract | Publisher Full Text | Free Full Text

29. Chen $X$, Zheng F, Qing Y, et al.: Epidemiological and clinical features of 291 cases with coronavirus disease 2019 in areas adjacent to Hubei, China: a double-center observational study. MedRxiv. 2020. Publisher Full Text

30. Chen X, Ling J, Mo P, et al.: Restoration of leukomonocyte counts is associated with viral clearance in COVID-19 hospitalized patients. MedRxiv. 2020.

Publisher Full Text

31. Duan Q, Guo G, Ren Y, et al.: Treatment Outcomes, Influence Factors of 116 Hospitalized COVID-19 Patients with Longer/Prolonged Treatment Course in Wuhan, China. Influence Factors. 2020; 116.

32. Gao Y, Li T, Han M, et al.: Diagnostic utility of clinical laboratory data determinations for patients with the severe COVID-19. J Med Virol. 2020; 92(7): 791-796.

PubMed Abstract | Publisher Full Text | Free Full Text

33. Guan W], Ni ZY, Hu Y, et al.: Clinical Characteristics of Coronavirus Disease 2019 in China. N EnglJ Med. 2020; 382(18): 1708-1720. PubMed Abstract | Publisher Full Text | Free Full Tex

34. Huang $C$, Wang $Y, L i X$, et al.: Clinical features of patients infected with 2019 novel coronavirus in Wuhan, China. Lancet. 2020; 395(10223): 497-506. PubMed Abstract | Publisher Full Text | Free Full Text
35. Jian-ya G: Clinical characteristics of $\mathbf{5 1}$ patients discharged from hospital with COVID-19 in Chongqing, China. medRxiv. 2020.

Publisher Full Text

36. Liu T, Zhang J, Yang Y, et al:: The potential role of IL-6 in monitoring coronavirus disease 2019. 2020.

Publisher Full Text

37. Shi Q, Zhao K, Yu J, et al.: Clinical characteristics of 101 non-surviving hospitalized patients with COVID-19: A single center, retrospective study. medRxiv. 2020.

Reference Source

38. Wang D, Hu B, Hu C, et al.: Clinical Characteristics of $\mathbf{1 3 8}$ Hospitalized Patients With 2019 Novel Coronavirus-Infected Pneumonia in Wuhan China. JAMA. 2020; 323(11): 1061-1069.

PubMed Abstract | Publisher Full Text | Free Full Text

39. Wang $\mathrm{H}$, Luo $\mathrm{S}$, Shen $\mathrm{Y}$, et al.: Multiple enzyme release, inflammation storm and hypercoagulability are prominent indicators for disease progression in COVID-19: a multi-centered, correlation study with CT imaging score. 2020. Publisher Full Text

40. Xu Y, Li Y-r, Zeng Q, et al.: Clinical characteristics of SARS-CoV-2 pneumonia compared to controls in Chinese Han population. medRxiv. 2020. Publisher Full Text

41. Zhang Jj, Dong $X$, Cao YY, et al.: Clinical characteristics of 140 patients infected with SARS-CoV-2 in Wuhan, China. Allergy. 2020; 75(7): 1730-1741. PubMed Abstract | Publisher Full Text

42. Zhou F, Yu T, Du R, et al.: Clinical course and risk factors for mortality of adult inpatients with COVID-19 in Wuhan, China: a retrospective cohort study. Lancet. 2020; 395(10229): 1054-1062.

PubMed Abstract | Publisher Full Text | Free Full Text

43. Li J, He X, Zhang W, et al.: Meta-analysis investigating the relationship between clinical features, outcomes, and severity of severe acute respiratory syndrome coronavirus 2 (SARS-CoV-2) pneumonia. Am J Infect Control. 2020; S0196-6553(20)30369-2. PubMed Abstract | Publisher Full Text | Free Full Text

44. Yang J, Zheng $Y$, Gou X, et al.: Prevalence of comorbidities and its effects in patients infected with SARS-CoV-2: a systematic review and meta-analysis. Int J Infect Dis. 2020; 94: 91-95.

PubMed Abstract | Publisher Full Text | Free Full Text

45. Badawi A, Velummailum R, Ryoo SG, et al.: Prevalence of chronic comorbidities in dengue fever and West Nile virus: A systematic review and meta-analysis. PLoS One. 2018; 13(7): e0200200. PubMed Abstract | Publisher Full Text | Free Full Text

46. Vasdev S, Stuckless J, Richardson V: Role of the immune system in hypertension: modulation by dietary antioxidants. Int J Angiol. 2011; 20(4): 189-212.

PubMed Abstract | Publisher Full Text | Free Full Text

47. Ferlita S, Yegiazaryan A, Noori N, et al.: Type $\mathbf{2}$ Diabetes Mellitus and Altered Immune System Leading to Susceptibility to Pathogens, Especially Mycobacterium tuberculosis. J Clin Med. 2019; 8(12): 2219. PubMed Abstract | Publisher Full Text | Free Full Text

48. Lazzerini PE, Hamilton RM, Boutjdir M: Editorial: Cardioimmunology: Inflammation and Immunity in Cardiovascular Disease. Front Cardiovasc Med. 2019; 6: 181.

PubMed Abstract | Publisher Full Text | Free Full Text

49. Perlman S, Dandekar AA: Immunopathogenesis of coronavirus infections: implications for SARS. Nat Rev Immunol. 2005; 5(12): 917-27.

PubMed Abstract | Publisher Full Text | Free Full Text

50. Green SJ: Covid-19 accelerates endothelial dysfunction and nitric oxide deficiency. Microbes Infect. 2020; 22(4-5): 149-150. PubMed Abstract | Publisher Full Text | Free Full Text

51. Russell CD, Parajuli A, Gale HJ, et al.: The utility of peripheral blood leucocyte ratios as biomarkers in infectious diseases: A systematic review and metaanalysis. J Infect. 2019; 78(5): 339-348.

PubMed Abstract | Publisher Full Text | Free Full Text

52. Pascutti MF, Erkelens MN, Nolte MA: Impact of Viral Infections on Hematopoiesis: From Beneficial to Detrimental Effects on Bone Marrow Output. Front Immunol. 2016; 7: 364.

PubMed Abstract | Publisher Full Text | Free Full Text

53. Li Y, Wang H, Wang F, et al.: Comparison of Hospitalized Patients with pneumonia caused by COVID-19 and influenza $A$ in children under 5 years. Int J Infect Dis. 2020; 98: 80-83.

PubMed Abstract | Publisher Full Text | Free Full Text

54. Coperchini F, Chiovato L, Croce L, et al:: The cytokine storm in COVID-19: An overview of the involvement of the chemokine/chemokine-receptor system. Cytokine Growth Factor Rev. 2020; 53: 25-32. PubMed Abstract | Publisher Full Text | Free Full Text

55. Quartuccio L, Sonaglia A, McGonagle D, et al:: Profiling CoviD-19 pneumonia progressing into the cytokine storm syndrome: results from a single Italian Centre study on tocilizumab versus standard of care. J Clin Virol. 2020; 129: 104444.

PubMed Abstract | Publisher Full Text | Free Full Text

56. Papayannopoulos V: Neutrophil extracellular traps in immunity and disease. Nat Rev Immunol. 2018; 18(2): 134-147.

PubMed Abstract | Publisher Full Text 
57. Mikacenic C, Moore R, Dmyterko V, et al.: Neutrophil extracellular traps (NETs) are increased in the alveolar spaces of patients with ventilatorassociated pneumonia. Crit Care. 2018; 22(1): 358. PubMed Abstract | Publisher Full Text | Free Full Text

58. Koyasu S, Moro K: Role of innate lymphocytes in infection and inflammation. Front Immunol. 2012; 3: 101. PubMed Abstract | Publisher Full Text | Free Full Text

59. Liu M, Wang T, Zhou Y, et al.: Potential Role of ACE2 in Coronavirus Disease 2019 (COVID-19) Prevention and Management. J Transl Int Med. 2020; 8(1): 9-19.

PubMed Abstract | Publisher Full Text | Free Full Text

60. To KF, Lo AW: Exploring the pathogenesis of severe acute respiratory syndrome (SARS): the tissue distribution of the coronavirus (SARS-CoV) and its putative receptor, angiotensin-converting enzyme 2 (ACE2). J Pathol. 2004; 203(3): 740-3.

PubMed Abstract | Publisher Full Text | Free Full Text

61. Shachar I, Karin N: The dual roles of inflammatory cytokines and chemokines in the regulation of autoimmune diseases and their clinica implications. J Leukoc Biol. 2013; 93(1): 51-61.

PubMed Abstract | Publisher Full Text

62. Hamming I, Timens W, Bulthuis M, et al.: Tissue distribution of ACE2 protein, the functional receptor for SARS coronavirus. A first step in understanding SARS pathogenesis.J Pathol. 2004; 203(2): 631-7. PubMed Abstract | Publisher Full Text | Free Full Text

63. Fischer $\mathrm{K}$, Hoffmann $\mathrm{P}$, Voelkl S, et al.: Inhibitory effect of tumor cell-derived lactic acid on human T cells. Blood. 2007; 109(9): 3812-9. PubMed Abstract | Publisher Full Text

64. Chhetri S, Khamis F, Pandak N, et al.: A fatal case of COVID-19 due to metabolic acidosis following dysregulate inflammatory response (cytokine storm). IDCases. 2020; 21: e00829.

PubMed Abstract | Publisher Full Text | Free Full Text

65. Levi M, Keller TT, van Gorp E, et al.: Infection and inflammation and the coagulation system. Cardiovasc Res. 2003; 60(1): 26-39. PubMed Abstract | Publisher Full Text

66. Lovren F, Pan $Y$, Quan A, et al.: Angiotensin converting enzyme-2 confers endothelial protection and attenuates atherosclerosis. Am J Physiol Heart Circ Physiol. 2008; 295(4): H1377-84. PubMed Abstract | Publisher Full Text

67. Varga Z, Flammer AJ, Steiger $P$, et al.: Endothelial cell infection and endotheliitis in COVID-19. Lancet. 2020; 395(10234): 1417-1418. PubMed Abstract | Publisher Full Text | Free Full Text

68. Marietta M, Ageno W, Artoni A, et al.: COVID-19 and haemostasis: a position paper from Italian Society on Thrombosis and Haemostasis (SISET). Blood Transfus. 2020; 18(3): 167-169.

PubMed Abstract | Publisher Full Text | Free Full Text

69. Becker RC: COVID-19 update: Covid-19-associated coagulopathy.J Thromb Thrombolysis. 2020; 50(1): 54-67.

PubMed Abstract | Publisher Full Text | Free Full Text

70. Matsuo T, Kobayashi H, Kario K, et al.: Fibrin D-dimer in thrombogenic disorders. Semin Thromb Hemost. 2000; 26(1): 101-7. PubMed Abstract | Publisher Full Text

71. Khan IH, Savarimuthu S, Leung MST, et al.: The need to manage the risk of thromboembolism in COVID-19 patients. J Vasc Surg. 2020; 72(3): 799-804. PubMed Abstract | Publisher Full Text | Free Full Text

72. Garcia-Olive I, Sintes H, Radua J, et al.: D-dimer in patients infected with COVID-19 and suspected pulmonary embolism. Respir Med. 2020; 169: 106023.

PubMed Abstract | Publisher Full Text | Free Full Text

73. Ullah W, Saeed R, Sarwar U, et al.: COVID-19 complicated by Acute Pulmonary Embolism and Right-Sided Heart Failure. JACC Case Rep. 2020; 2(9): 1379-1382.

PubMed Abstract | Publisher Full Text | Free Full Text

74. Nahum J, Morichau-Beauchant T, Daviaud F, et al.: Venous Thrombosis Among Critically Ill Patients With Coronavirus Disease 2019 (COVID-19). JAMA Netw Open. 2020; 3(5): e2010478.

PubMed Abstract | Publisher Full Text | Free Full Text

75. Rodriguez-Morales AJ, Cardona-Ospina JA, Gutierrez-Ocampo E, et al.: Clinical, laboratory and imaging features of COVID-19: A systematic review and meta-analysis. Travel Med Infect Dis. 2020; 34: 101623. PubMed Abstract | Publisher Full Text | Free Full Text

76. Aziz M, Fatima R, Assaly R: Elevated interleukin-6 and severe CovID-19: A meta-analysis. J Med Virol. 2020.

PubMed Abstract | Publisher Full Text | Free Full Text

77. Zaim S, Chong JH, Sankaranarayanan V, et al.: COVID-19 and Multiorgan Response. Curr Probl Cardiol. 2020; 45(8): 100618. PubMed Abstract | Publisher Full Text | Free Full Text

78. Yang $X$, Jin $Y$, Li R, et al.: Prevalence and impact of acute renal impairment on COVID-19: a systematic review and meta-analysis. Crit Care. 2020; 24(1): 356. PubMed Abstract | Publisher Full Text | Free Full Text

79. Chen YT, Shao SC, Hsu CK, et al.: Incidence of acute kidney injury in CovID-19 infection: a systematic review and meta-analysis. Crit Care. 2020; 24(1): 346. PubMed Abstract | Publisher Full Text | Free Full Text

80. Rossi GM, Delsante M, Pilato FP, et al.: Kidney biopsy findings in a critically ill COVID-19 patient with dialysis-dependent acute kidney injury: a case against "SARS-CoV-2 nephropathy". Kidney Int Rep. 2020; 5(7): 1100-1105. PubMed Abstract | Publisher Full Text | Free Full Text

81. Harapan H, Itoh N, Yufika A, et al.: Coronavirus disease 2019 (COVID-19): A literature review. J Infect Public Health. 2020; 13(5): 667-673. PubMed Abstract | Publisher Full Text | Free Full Text

82. Batlle D, Soler MJ, Sparks MA, et al.: Acute kidney injury in CovID-19: emerging evidence of a distinct pathophysiology. J Am Soc Nephrol. 2020 31(7): 1380-1383.

PubMed Abstract | Publisher Full Text | Free Full Text

83. Nasr SH, Kopp JB: COVID-19-Associated Collapsing Glomerulopathy: An Emerging Entity. Kidney Int Rep. 2020; 5(6): 759-761. PubMed Abstract | Publisher Full Text | Free Full Text

84. Henry BM, Vikse J, Benoit S, et al.: Hyperinflammation and derangement of renin-angiotensin-aldosterone system in COVID-19: A novel hypothesis for clinically suspected hypercoagulopathy and microvascular immunothrombosis. Clin Chim Acta. 2020; 507: 167-173. PubMed Abstract | Publisher Full Text | Free Full Text

85. Kai H, Kai M: Interactions of coronaviruses with ACE2, angiotensin II, and RAS inhibitors-lessons from available evidence and insights into COVID-19. Hypertens Res. 2020; 43(7): 648-654.

PubMed Abstract | Publisher Full Text | Free Full Text

86. Chai $X$, Hu L, Zhang $Y$, et al.: Specific ACE2 expression in cholangiocytes may cause liver damage after 2019-nCoV infection. bioRxiv. 2020. Publisher Full Text

87. $\mathrm{Xu} \mathrm{Z}$, Shi L, Wang $Y$, et al.: Pathological findings of COVID-19 associated with acute respiratory distress syndrome. Lancet Respir Med. 2020; 8(4): 420-422. PubMed Abstract | Publisher Full Text | Free Full Text

88. Park JH, Jang W, Kim SW, et al.: The Clinical Manifestations and Chest Computed Tomography Findings of Coronavirus Disease 2019 (COVID-19) Patients in China: A Proportion Meta-Analysis. Clin Exp Otorhinolaryngol. 2020; 13(2): 95-105.

PubMed Abstract | Publisher Full Text | Free Full Text

89. Zhang ZL, Hou YL, Li DT, et al.: Laboratory findings of COVID-19: a systematic review and meta-analysis. Scand J Clin Lab Invest. 2020; 1-7. PubMed Abstract | Publisher Full Text | Free Full Text

90. Jain V, Yuan JM: Predictive symptoms and comorbidities for severe COVID-19 and intensive care unit admission: a systematic review and meta-analysis. Int J Public Health. 2020; 1-14.

PubMed Abstract | Publisher Full Text | Free Full Text

91. Li LQ, Huang T, Wang YQ, et al.: COVID-19 patients' clinical characteristics, discharge rate, and fatality rate of meta-analysis. J Med Virol. 2020; 92(6): 577-583.

PubMed Abstract | Publisher Full Text | Free Full Text

92. Tenforde MW, Kim SS, Lindsell CJ, et al.: Symptom Duration and Risk Factors for Delayed Return to Usual Health Among Outpatients with COVID-19 in a Multistate Health Care Systems Network - United States, March-June 2020. MMWR Morb Mortal Wkly Rep. 2020; 69(30): 993-998.

PubMed Abstract | Publisher Full Text | Free Full Text

93. Mudatsir M, Fajar ]: PRISMA CHECKLIST FOR "Predictors of COVID-19 severity: a systematic review and meta-analysis". figshare. Media. 2020. http://www.doi.org/10.6084/m9.figshare.12813683.v1 


\section{Open Peer Review}

\section{Current Peer Review Status:}

\section{Version 1}

Reviewer Report 02 November 2020

https://doi.org/10.5256/f1000research.28897.r72568

(C) 2020 Wilder-Smith A. This is an open access peer review report distributed under the terms of the Creative Commons Attribution License, which permits unrestricted use, distribution, and reproduction in any medium, provided the original work is properly cited.

\section{Annelies Wilder-Smith}

Heidelberg Institute of Global Health, University of Heidelberg, Heidelberg, Germany

The strength of this paper is the meta-analysis in terms of effect estimates. The weakness is the focus of data from China, while we should learn more from global data including the comparison between HIC and LMIC.

In China, severity was also found to correlate with the force of infection, eg those in high transmission areas had more severe disease outcomes than those from lower transmission areas in China, see: Exposure to SARS-CoV-2 in a high transmission setting increases the risk of severe COVID-19 compared with exposure to a low transmission setting?

Chen D, Hu C, Su F, Song Q, Wang Z. J Travel Med. 2020 Aug 20;27(5):taaa094. doi: 10.1093/jtm/taaa094.

The authors highlight the need for a scoring system for the prediction of severity. There is another reason why it is important to identify risk factors for severe disease: to guide prioritization of high risk target populations for vaccination

\section{References}

1. Chen D, Hu C, Su F, Song Q, et al.: Exposure to SARS-CoV-2 in a high transmission setting increases the risk of severe COVID-19 compared with exposure to a low transmission setting?. Journal of Travel Medicine. 2020; 27 (5). Publisher Full Text

Are the rationale for, and objectives of, the Systematic Review clearly stated? Yes

Are sufficient details of the methods and analysis provided to allow replication by others? Yes

Is the statistical analysis and its interpretation appropriate? Yes 
Are the conclusions drawn adequately supported by the results presented in the review? Yes

Competing Interests: No competing interests were disclosed.

Reviewer Expertise: COVID-19, Zika and dengue

I confirm that I have read this submission and believe that I have an appropriate level of expertise to confirm that it is of an acceptable scientific standard.

Author Response 03 Nov 2020

Mudatsir Mudatsir

Response to comments from reviewers:

Reviewer 2\#

1. The strength of this paper is the meta-analysis in terms of effect estimates. The weakness is the focus of data from China, while we should learn more from global data including the comparison between HIC and LMIC. In China, severity was also found to correlate with the force of infection, eg those in high transmission areas had more severe disease outcomes than those from lower transmission areas in China, see: Exposure to SARS-CoV-2 in a high transmission setting increases the risk of severe COVID-19 compared with exposure to a low transmission setting? Chen D, Hu C, Su F, Song Q, Wang Z. J Travel Med. 2020 Aug 20;27(5):taaa094. doi: 10.1093/jtm/taaa094.1

Response: The additional limitation has been added, as suggested

2. The authors highlight the need for a scoring system for the prediction of severity. There is another reason why it is important to identify risk factors for severe disease: to guide prioritization of high risk target populations for vaccination.

Response: The additional clinical implication has been added, as suggested.

Competing Interests: We have no competing interest.

Reviewer Report 21 September 2020

https://doi.org/10.5256/f1000research.28897.r71054

(C) 2020 Arab-Zozani M. This is an open access peer review report distributed under the terms of the Creative Commons Attribution License, which permits unrestricted use, distribution, and reproduction in any medium, provided the original work is properly cited.

\section{Morteza Arab-Zozani}

Social Determinants of Health Research Center, Birjand University of Medical Sciences, Birjand, Iran

In this meta-analysis, you investigated the predictors of COVID-19 severity through the literature. 
You considered a topic of interest and provided a well-written manuscript. However, there are some things that will improve your reporting.

Abstract, method section, please insert detail about critical/quality appraisal of the included studies.

Abstract, method section, line 1, please remove " and extracted" from the text. It maybe causes a misunderstanding between this step and the data extraction step.

Method section, please remove line five. "the protocols for the ...". Mentioning the PRISMA is enough.

Method section, eligibility criteria, (2) please mention the type of data for adequate data. what is adequate data?

Method section, search strategy, why is Scopus not searched? You may have missed some articles that are only indexed in Scopus.

Method section, search strategy, this sentence not related to this section. If you limit the search to EN publication then you need to change the verb. If not this sentence related to inclusion criteria.

Method section, search strategy, based on PRISMA, add at least one search strategy for one database as a supplement.

Method section, data extraction, please added the country of origin for each study. The predictors may be different from one setting to another setting.

Method section, data extraction, please add details about how resolved disagreement between reviewers.

Method section, how did you handle the publication bias?

Result section, there is some problem in figure 1. Please fill it considering other related studies. The number for "record screened" is incorrect.

Result section, table 1, all studies are from China. If all studies are from China it is better to change the title. these are a predictor of severity in China. In my opinion, this is a limitation of your study.

\section{Cheers}

\section{Are the rationale for, and objectives of, the Systematic Review clearly stated? Yes}

Are sufficient details of the methods and analysis provided to allow replication by others? Partly

Is the statistical analysis and its interpretation appropriate? 
Yes

Are the conclusions drawn adequately supported by the results presented in the review? Yes

Competing Interests: No competing interests were disclosed.

Reviewer Expertise: Systematic review and meta-analysis in health and medical intervention

I confirm that I have read this submission and believe that I have an appropriate level of expertise to confirm that it is of an acceptable scientific standard.

Author Response 03 Nov 2020

Mudatsir Mudatsir

Response to comments from reviewers:

Reviewer 1\#

1. In this meta-analysis, you investigated the predictors of COVID-19 severity through the literature. You considered a topic of interest and provided a well-written manuscript.

However, there are some things that will improve your reporting. Abstract, method section, please insert detail about critical/quality appraisal of the included studies.

Response: The description of the quality assessment of included papers has been added in the method of abstract.

2. Abstract, method section, line 1, please remove " and extracted" from the text. It maybe causes a misunderstanding between this step and the data extraction step.

Response: We have removed "and extracted".

3. Method section, please remove line five. "the protocols for the ...". Mentioning the PRISMA is enough.

Response: PRISMA checklist may be interpreted as the general guideline in meta-analysis. The specific protocols may differ among meta-analysis studies; for example, the protocols of meta-analysis in gene polymorphism may differ from the protocols of meta-analysis in risk factors identification. In our manuscript, we referred to previous meta-analysis studies in the context of risk factors identification.

4. Method section, eligibility criteria, (2) please mention the type of data for adequate data. what is adequate data?

Response: The additional information related to adequate data has been provided.

5. Method section, search strategy, why is Scopus not searched? You may have missed some articles that are only indexed in Scopus.

Response: We also performed the searching strategy in Scopus as of 5 April 2020, however, we did not find additional articles.

6. Method section, search strategy, this sentence not related to this section. If you limit the search to EN publication then you need to change the verb. If not this sentence related to 
inclusion criteria. Response: English publication language has been added to eligibility criteria.

7. Method section, search strategy, based on PRISMA, add at least one search strategy for one database as a supplement.

Response: The additional database has been added as the additional database.

8. Method section, data extraction, please added the country of origin for each study. The predictors may be different from one setting to another setting.

Response: Country of origin has been added in data extraction.

9. Method section, data extraction, please add details about how resolved disagreement between reviewers.

Response: The additional sentence has been added to describe how to resolve the disagreement.

10. Method section, how did you handle the publication bias?

Response: The assessment of publication bias has been described in statistical analysis using Egger test. In the results, we presented in Tables 2 \& 3.

11. Result section, there is some problem in figure 1. Please fill it considering other related studies. The number for "record screened" is incorrect.

Response: In Figure 1, we used the template from PRISMA for the article selection pathway.

12. Result section, table 1, all studies are from China. If all studies are from China it is better to change the title. these are a predictor of severity in China. In my opinion, this is a limitation of your study.

Response: We tried to search articles in all regions, however, at the time frame of our searching, we only found articles in China.

Competing Interests: We have no competing interest. 
The benefits of publishing with F1000Research:

- Your article is published within days, with no editorial bias

- You can publish traditional articles, null/negative results, case reports, data notes and more

- The peer review process is transparent and collaborative

- Your article is indexed in PubMed after passing peer review

- Dedicated customer support at every stage

For pre-submission enquiries, contact research@f1000.com 